\title{
QUASILINEAR EQUATIONS WITH NATURAL GROWTH IN THE GRADIENTS IN SPACES OF SOBOLEV MULTIPLIERS
}

\author{
KARTHIK ADIMURTHI ${ }^{1}$ AND NGUYEN CONG PHUC ${ }^{2}$
}

\begin{abstract}
We study the existence problem for a class of nonlinear elliptic equations whose prototype is of the form $-\Delta_{p} u=|\nabla u|^{p}+\sigma$ in a bounded domain $\Omega \subset \mathbb{R}^{n}$. Here $\Delta_{p}, p>1$, is the standard $p$-Laplacian operator defined by $\Delta_{p} u=\operatorname{div}\left(|\nabla u|^{p-2} \nabla u\right)$, and the datum $\sigma$ is a signed distribution in $\Omega$. The class of solutions that we are interested in consists of functions $u \in W_{0}^{1, p}(\Omega)$ such that $|\nabla u| \in M\left(W^{1, p}(\Omega) \rightarrow L^{p}(\Omega)\right)$, a space pointwise Sobolev multipliers consisting of functions $f \in L^{p}(\Omega)$ such that

$$
\int_{\Omega}|f|^{p}|\varphi|^{p} d x \leq C \int_{\Omega}\left(|\nabla \varphi|^{p}+|\varphi|^{p}\right) d x \quad \forall \varphi \in C^{\infty}(\Omega),
$$

for some $C>0$. This is a natural class of solutions at least when the distribution $\sigma$ is nonnegative and compactly supported in $\Omega$. We show essentially that, with only a gap in the smallness constants, the above equation has a solution in this class if and only if one can write $\sigma=\operatorname{div} F$ for a vector field $F$ such that $|F|^{\frac{1}{p-1}} \in M\left(W^{1, p}(\Omega) \rightarrow L^{p}(\Omega)\right)$.

As an important application, via the exponential transformation $u \mapsto v=e^{\frac{u}{p-1}}$, we obtain an existence result for the quasilinear equation of Schrödinger type $-\Delta_{p} v=\sigma v^{p-1}$, $v \geq 0$ in $\Omega$, and $v=1$ on $\partial \Omega$, which is interesting in its own right.
\end{abstract}

\section{INTRODUCTION}

In this work, we study the existence problem for the quasilinear elliptic equation

$$
\begin{cases}-\operatorname{div} \mathcal{A}(x, \nabla u)=\mathcal{B}(x, u, \nabla u)+\sigma & \text { in } \quad \Omega \\ u=0 & \text { on } \partial \Omega\end{cases}
$$

in a bounded domain $\Omega \subset \mathbb{R}^{n}$, where the principal operator $\operatorname{div} \mathcal{A}(x, u, \nabla u)$ is a Leray-Lions operator defined on $W_{0}^{1, p}(\Omega)$ and $|\mathcal{B}(x, u, \nabla u)| \lesssim|\nabla u|^{p}, p>1$. Precise assumptions on the domain $\Omega$ and the nonlinearities $\mathcal{A}, B$ will be made explicitly later. Here the 'datum' $\sigma$ is a general distribution in $\Omega$, and $W_{0}^{1, p}(\Omega)$ is defined as the completion of $C_{c}^{\infty}(\Omega)$ under the semi-norm $\|\nabla(\cdot)\|_{L^{p}(\Omega)}$.

\footnotetext{
${ }^{1}$ Supported in part by National Research Foundation of Korea grant funded by the Korean government (MEST) (NRF-2015R1A2A1A15053024).

${ }^{2}$ Supported in part by Simons Foundation, award number 426071.
} 
A typical example of (1.1) after which it is modeled is the following quasilinear elliptic equations with gradient nonlinearity of natural growth of the form

$$
-\Delta_{p} u=|\nabla u|^{p}+\sigma \text { in } \Omega, \quad u=0 \text { on } \partial \Omega,
$$

where $\Delta_{p} u:=\operatorname{div}\left(|\nabla u|^{p-2} \nabla u\right), p>1$, is the $p$-Laplacian operator.

When $p=2$, equation (1.2) becomes a stationary viscous Hamilton-Jacobi equation, also known as the Kardar-Parisi-Zhang equation that appears in the physical theory of growth and roughening of surfaces $[21,22]$. Moreover, via the transformation $u \mapsto v:=e^{\frac{u}{p-1}}$, this equation can be transformed into the Schrödinger type equation

$$
-\Delta_{p} v=(p-1)^{1-p} \sigma v^{p-1} \text { in } \Omega, \quad v \geq 0 \text { in } \Omega, \quad v=1 \text { on } \partial \Omega,
$$

a connection that we shall discuss at the end of this section.

When it comes to the existence theory, it is well-known that in order for (1.2) to have a solution the datum $\sigma$ must be both small and regular enough. For example, if $\sigma$ is a nonnegative locally finite measure in $\Omega$ and the first equation in (1.2) has a $W_{\text {loc }}^{1, p}(\Omega)$ solution (without any boundary condition), then $\sigma$ must obey the weighted Poincaré-Sobolev inequality (see $[17,18,19])$ :

$$
\int_{\Omega}|\varphi|^{p} d \sigma \leq(p-1)^{p-1} \int_{\Omega}|\nabla \varphi|^{p} d x \quad \forall \varphi \in C_{c}^{\infty}(\Omega) .
$$

Moreover, when $\sigma \geq 0$ the nonlinear term $|\nabla u|^{p}$ also obeys a similar weighted inequality

$$
\int_{\Omega}|\varphi|^{p}|\nabla u|^{p} d x \leq p^{p} \int_{\Omega}|\nabla \varphi|^{p} d x \quad \forall \varphi \in C_{c}^{\infty}(\Omega) .
$$

If we assume in addition that $\operatorname{supp}(\sigma)=K$ where $K$ is a compact set in $\Omega$, then by multiplying by a cutoff function $\chi \in C_{c}^{\infty}(\Omega), 0 \leq \chi \leq 1$, and $\chi=1$ on $K$, we see from (1.3) that

$$
\int_{\Omega}|\varphi|^{p} d \sigma \leq \lambda \int_{\mathbb{R}^{n}}\left(|\nabla \varphi|^{p}+|\varphi|^{p}\right) d x \quad \forall \varphi \in C_{c}^{\infty}\left(\mathbb{R}^{n}\right)
$$

with a constant $\lambda>0$. Note that the 'test functions' $\varphi$ in (1.5) are now allowed to have support not contained in $\Omega$. However, in general from (1.4) we cannot say that $|\nabla u|^{p}$ obeys the similar inequality

$$
\int_{\Omega}|\varphi|^{p}|\nabla u|^{p} d x \leq A \int_{\mathbb{R}^{n}}\left(|\nabla \varphi|^{p}+|\varphi|^{p}\right) d x \quad \forall \varphi \in C_{c}^{\infty}\left(\mathbb{R}^{n}\right),
$$


for some $A>0$, not even when $u \in W_{0}^{1, p}(\Omega)$. The main difference between (1.4) and (1.6) lies in the behavior of $|\nabla u|^{p}$ near the boundary of $\Omega$. In contrast to (1.4), inequality (1.6) requires that $|\nabla u|^{p}$ have stronger regularity up to the boundary of $\Omega$.

In this paper, we only insist on obtaining solutions to (1.1) that belong to the class $\mathcal{C}$ of functions $u \in W_{0}^{1, p}(\Omega)$ such that inequality (1.6) holds with some $A>0$. Our goal is to find the largest space $\mathcal{F}$ of data on $\Omega$ so that whenever $\sigma \in \mathcal{F}$ with a sufficiently small norm then (1.1) has a solution in $\mathcal{C}$. In brief, our main result states that, with only a gap in the smallness constants, equation (1.1) has a solution in the class $\mathcal{C}$ if and only if the distribution $\sigma$ can be written in the form $\sigma=\operatorname{div} F$ for a vector field $F \in L^{\frac{p}{p-1}}\left(\Omega, \mathbb{R}^{n}\right)$ such that

$$
\int_{\Omega}|F|^{\frac{p}{p-1}}|\varphi|^{p} d x \leq \lambda \int_{\mathbb{R}^{n}}\left(|\nabla \varphi|^{p}+|\varphi|^{p}\right) d x \quad \forall \varphi \in C_{c}^{\infty}\left(\mathbb{R}^{n}\right)
$$

for some $\lambda>0$. For the simpler equation (1.2) on, say, $C^{1}$ domains our results read as follows.

Theorem 1.1. (i) Suppose that (1.2) has a solution in $u \in W_{0}^{1, p}(\Omega)$ such that (1.6) holds for some $A>0$ then it is necessary that $\sigma=\operatorname{div} F$ for a vector field $F \in L^{\frac{p}{p-1}}\left(\Omega, \mathbb{R}^{n}\right)$ such that (1.7) holds with a $\lambda>0$.

(ii) Conversely, suppose that $\Omega$ is a bounded $C^{1}$ domain. Then there exists a constant $\lambda_{0}=\lambda_{0}(n, p, \Omega)>0$ such that if $\sigma=\operatorname{div} F$ for a vector field $F$ satisfying (1.7) with some $\lambda \in\left(0, \lambda_{0}\right]$, then (1.2) has a solution $u \in W_{0}^{1, p}(\Omega)$ satisfying the weighted inequality (1.6) for some $A>0$.

The condition (1.7) simply means that the function $\mid F^{\frac{1}{p-1}} \chi_{\Omega}$ belongs to the space of Sobolev multipliers $M\left(W^{1, p}\left(\mathbb{R}^{n}\right) \rightarrow L^{p}\left(\mathbb{R}^{n}\right)\right)$, which consists of functions $f \in L_{\text {loc }}^{p}\left(\mathbb{R}^{n}\right)$ such that

$$
\int_{\mathbb{R}^{n}}|f|^{p}|\varphi|^{p} d x \leq C \int_{\mathbb{R}^{n}}\left(|\nabla \varphi|^{p}+|\varphi|^{p}\right) d x \quad \forall \varphi \in C_{c}^{\infty}\left(\mathbb{R}^{n}\right),
$$

for some $C>0$. The norm of such $f$ is the $p$-th root of the best constant $C$ in the above inequality.

For our purpose, we denote by $\mathrm{M}^{1, p}(\Omega)$ the space of functions $f \in L^{p}(\Omega)$ such that $f \chi_{\Omega} \in M\left(W^{1, p}\left(\mathbb{R}^{n}\right) \rightarrow L^{p}\left(\mathbb{R}^{n}\right)\right)$ with norm $\|f\|_{\mathrm{M}^{1, p}(\Omega)}:=\left\|f \chi_{\Omega}\right\|_{M\left(W^{1, p}\left(\mathbb{R}^{n}\right) \rightarrow L^{p}\left(\mathbb{R}^{n}\right)\right)}$. The space $\mathrm{M}^{1, p}(\Omega)$ can also be described using the capacity associated to the Sobolev space $W^{1, p}\left(\mathbb{R}^{n}\right)$; see Section 2 below. Moreover, it is known that for any bounded Lipschitz 
domain $\Omega$, the space $\mathrm{M}^{1, p}(\Omega)$ coincides with the multiplier space $M\left(W^{1, p}(\Omega) \rightarrow L^{p}(\Omega)\right)$, which consists of functions $f \in L^{p}(\Omega)$ such that

$$
\int_{\Omega}|f|^{p}|\varphi|^{p} d x \leq C \int_{\Omega}\left(|\nabla \varphi|^{p}+|\varphi|^{p}\right) d x \quad \forall \varphi \in C^{\infty}(\Omega)
$$

for some $C>0$; see [27, Theorem 9.3.1].

It is worth pointing out, as we show in Theorem 3.1 below, that the solution $u$ obtained in Theorem 1.1(ii) obeys a stability estimate

$$
\||\nabla u|\|_{\mathrm{M}^{1, p}(\Omega)} \leq T_{0}\left\||F|^{\frac{1}{p-1}}\right\|_{\mathrm{M}^{1, p}(\Omega)}
$$

for a constant $T_{0}>0$. Moreover, we have $e^{\mu|u|}-1 \in W_{0}^{1, p}(\Omega)$ provided $\mu \in\left[0, \mu_{0}\right]$ where $\mu_{0}=C\left\||F|^{\frac{1}{p-1}}\right\|_{\mathrm{M}^{1, p}(\Omega)}^{-1}$ for some $C>0$. In particular, the solution here is zero whenever $\sigma=0$. Note that, even for $\sigma=0$, in general $W_{0}^{1, p}(\Omega)$ solutions to (1.2) are not unique; see [12, Remark 2.11].

An existence criterion in the spirit of Theorem 1.1 also holds for equation (1.1) under quite general assumptions on $\mathcal{A}, \mathcal{B}$ and $\Omega$. In particular, $\mathcal{A}(x, \xi)$ could be discontinuous in the $x$-variable and $B$ could include a zero order term. Moreover, $\Omega$ could be irregular and include certain Lipschitz or fractal domains. These assumptions will be made precise in the next section. The result for equation (1.1), which is the main result of the paper and includes Theorem 1.1 as a special case, will be treated in Section 3 (see Theorem 3.1 below).

We next have the following remarks.

Remark 1.2. Let $\widetilde{\Omega}$ be another bounded open set such that $\Omega \Subset \widetilde{\Omega}$. Then by Poincaré's inequality we see that (1.7) is also equivalent to the homogeneous inequality

$$
\int_{\Omega}|F|^{\frac{p}{p-1}}|\varphi|^{p} d x \leq \lambda \int_{\widetilde{\Omega}}|\nabla \varphi|^{p} d x \quad \forall \varphi \in C_{c}^{\infty}(\widetilde{\Omega})
$$

for some $\lambda>0$. One also has similar statements for (1.5) and (1.6).

Remark 1.3. Let $\sigma$ be a nonnegative measure such that $\operatorname{supp}(\sigma) \Subset \Omega$. If the first equation in (1.2) has a distributional solution $u \in W_{\mathrm{loc}}^{1, p}(\Omega)$ (without any boundary condition), then it is still necessary that $\sigma=\operatorname{div} F$ with $|F|^{\frac{1}{p-1}} \in \mathrm{M}^{1, p}(\Omega)$. This follows from (1.5) and Lemma 2.6 below. This shows that the space $\mathrm{M}^{1, p}(\Omega)$ is quite natural for (1.2) at least for such data $\sigma$. 
We now mention some of the relevant results in the literature on the existence of $W_{0}^{1, p}(\Omega)$ solutions to $(1.2)$ or $(1.1)$. In $[11,12]$ an existence result in $W_{0}^{1, p}$ was obtained for small data $\sigma \in\left[W_{0}^{1, \frac{n}{n-p+1}}(\Omega)\right]^{*}$, i.e., $\sigma=\operatorname{div} F$ where $|F|^{\frac{1}{p-1}} \in L^{n}(\Omega)$ with a small norm. Later, it was shown in [13] that if $\sigma=\operatorname{div} F$ where $|F|^{\frac{1}{p-1}} \in L^{n, \infty}(\Omega)$ (the weak Lebesgue space) with a small norm than (1.1) admits a solution. Recently in [28], an existence result was obtained for $\sigma=\operatorname{div} F$ provided $|F|^{\frac{1}{p-1}}$ is small in $\mathcal{L}^{(1+\varepsilon) p,(1+\varepsilon) p}(\Omega)$ provided $0<\varepsilon<n / p-1$. Here $\mathcal{L}^{(1+\varepsilon) p,(1+\varepsilon) p}(\Omega), 0<\varepsilon<n / p-1$, is a Morrey space with norm given by

$$
\|f\|_{\mathcal{L}^{(1+\varepsilon) p,(1+\varepsilon) p(\Omega)}}^{(1+\varepsilon) p}=\sup \left[r^{(1+\varepsilon) p-n} \int_{B_{r}(z) \cap \Omega}|f|^{(1+\varepsilon) p} d x\right]
$$

where the supremum is taken over $z \in \Omega$ and $0<r \leq \operatorname{diam}(\Omega)$. Note that one has the following inclusions:

$$
L^{n}(\Omega) \subset L^{n, \infty}(\Omega) \subset \mathcal{L}^{(1+\varepsilon) p,(1+\varepsilon) p}(\Omega)
$$

provided $0<\varepsilon<n / p-1$. That $|F|^{\frac{1}{p-1}} \in \mathcal{L}^{(1+\varepsilon) p,(1+\varepsilon) p}(\Omega)$ implies inequality (1.7), i.e.,

$$
\mathcal{L}^{(1+\varepsilon) p,(1+\varepsilon) p}(\Omega) \subset \mathrm{M}^{1, p}(\Omega), \quad 0<\varepsilon<n / p-1,
$$

is well-known as it is a special case of the so-called Fefferman-Phong type conditions (see, e.g., $[8,9,10,29,31])$.

We note that there are also existence results obtained for (1.2) under weaker conditions on $\sigma$ and sometimes with sharp constants of smallness; see $[1,14,16]$ for nonnegative measure data and $[3,18,19]$ for distributional data. See also [11, 12, 13]. However, the solutions obtained in those papers may not behave very well at the boundary of $\Omega$, i.e., in general they do not satisfy inequality (1.6). See also the earlier work [17] where an existence result was obtained in the whole space $\Omega=\mathbb{R}^{n}$ in the 'linear' case $p=2$ for nonnegative measure data.

We now briefly describe the strategy that we use to construct a solution $u \in W_{0}^{1, p}(\Omega)$ of (1.2) such that $|\nabla u| \in \mathrm{M}^{1, p}(\Omega)$ under the assumption $\sigma=\operatorname{div} F$ where $|F|^{\frac{1}{p-1}} \in \mathrm{M}^{1, p}(\Omega)$ with a small norm. As in [12], we start with the approximate equation

$$
-\Delta_{p} u=\frac{|\nabla u|^{p}}{1+k^{-1}|\nabla u|^{p}}+\sigma \text { in } \Omega, \quad u=0 \text { on } \partial \Omega,
$$

where the parameter $k>0$ is to be sent to infinity eventually. Since $\sigma \in\left(W_{0}^{1, p}(\Omega)\right)^{*}$ and the first term on the right-hand side is uniformly bounded, by the theory of pseudomonotone operators (see, e.g., [25]), there exists a solution $u_{k} \in W_{0}^{1, p}(\Omega)$ to (1.8). However, this solution may not satisfy the property that $\left|\nabla u_{k}\right| \in \mathrm{M}^{1, p}(\Omega)$. Thus, to have this requiblack 
property for $u_{k}$ we have to construct it by a different way. As $\left\||F|^{\frac{1}{p-1}}\right\|_{\mathrm{M}^{1, p}(\Omega)}$ is small, it is natural to use Schauder's Fixed Point Theorem in a small ball of $\mathrm{M}^{1, p}(\Omega)$. The main difficulty in this approach is an a priori gradient estimate of the form

$$
\||\nabla u|\|_{\mathrm{M}^{1, p}(\Omega)} \leq C\left\||F|^{\frac{1}{p-1}}\right\|_{\mathrm{M}^{1, p}(\Omega)}
$$

for solutions $u$ to the basic equation

$$
-\Delta_{p} u=\operatorname{div} F \text { in } \Omega, \quad u=0 \text { on } \partial \Omega .
$$

Such a delicate gradient estimate can be obtained from an end-point weighted gradient estimate for (1.9) and has been prepablack in our earlier work [2]; see Lemma 2.7 below. Once solutions $\left\{u_{k}\right\}$ to (1.8) have been obtained with gradients being uniformly controlled in $\mathrm{M}^{1, p}(\Omega)$, the next step is to pass to the limit in (1.8), with $u_{k}$ in place of $u$, as $k \rightarrow \infty$. For that, it is enough to show the strong convergence of $\left\{u_{k}\right\}$ in $W_{0}^{1, p}(\Omega)$, a task that can be done via the truncation technique and appropriate test functions as in $[12,13]$. We mention that in our scenario this is possible since we have a uniform bound for $\left\{e^{\left|u_{k}\right|}-1\right\}$ in $W_{0}^{1, p}(\Omega)$, another important a priori estimate also obtained in Lemma 2.7.

To conclude this section, we discuss a connection of (1.2) and a Schrödinger type equation with distributional potential:

$$
-\Delta_{p} v=\sigma v^{p-1} \text { in } \Omega, \quad v \geq 0 \text { in } \Omega, \quad v=1 \text { on } \partial \Omega .
$$

This equation is interesting in its own right and its existence theory has been studied, e.g., in $[1,16,18,19]$. For $\sigma \in\left(W_{0}^{1, p}(\Omega)\right)^{*}$, by a solution of $(1.10)$ we mean a nonnegative function $v$ such that $v-1 \in W_{0}^{1, p}(\Omega), v^{p-1} \in W_{\text {loc }}^{1, p}(\Omega)$, and

$$
\int_{\Omega}|\nabla v|^{p-2} \nabla v \cdot \nabla \varphi d x=\left\langle\sigma, v^{p-1} \varphi\right\rangle \quad \forall \varphi \in C_{c}^{\infty}(\Omega) .
$$

Since $v^{p-1} \varphi \in W^{1, p}(\Omega)$ and $\operatorname{supp}\left(v^{p-1} \varphi\right) \Subset \Omega$, this definition makes sense even for $\sigma$ such that $\sigma \in\left(W_{0}^{1, p}\left(\Omega^{\prime}\right)\right)^{*}$ for any open set $\Omega^{\prime} \Subset \Omega$ (see $\left.[18,19]\right)$.

Formally, by using the transformation $u \mapsto v:=e^{\frac{u}{p-1}}$, the equation

$$
-\Delta_{p} u=|\nabla u|^{p}+(p-1)^{p-1} \sigma \text { in } \Omega, \quad u=0 \text { on } \partial \Omega,
$$

is transformed into (1.10). Indeed, using $\phi_{k}:=\varphi \min \left\{e^{u}, k\right\}, k>0, \varphi \in C_{c}^{\infty}(\Omega)$, as a test function for (1.11) and then letting $k \rightarrow \infty$ one can rigorously show from Theorem 3.1(ii) the following existence result for (1.10). 
Theorem 1.4. Let $\Omega$ be a bounded $C^{1}$ domain in $\mathbb{R}^{n}$. There exist $\lambda_{1}=\lambda_{1}(n, p, \Omega)>0$ and $T_{1}=T_{1}(n, p, \Omega)>0$ such that if $\sigma=\operatorname{div} F$ with $\left\||F|^{\frac{1}{p-1}}\right\|_{\mathrm{M}^{1, p}(\Omega)} \leq \lambda_{1}^{1 / p}$ then (1.10) has a nonnegative solution $v$ with $|\nabla \log (v)| \in \mathrm{M}^{1, p}(\Omega)$ and

$$
\||\nabla \log (v)|\|_{\mathrm{M}^{1, p}(\Omega)} \leq T_{1}\left\||F|^{\frac{1}{p-1}}\right\|_{\mathrm{M}^{1, p}(\Omega)} .
$$

Moreover, we have $v^{q}-1 \in W_{0}^{1, p}(\Omega)$ for all $q \in\left[0, \mu_{1}\right]$ where

$$
\mu_{1}=C_{1}(n, p, \Omega)\left\||F|^{\frac{1}{p-1}}\right\|_{\mathrm{M}^{1, p}(\Omega)}^{-1} \geq \max \{1, p-1\} .
$$

\section{Assumptions on $\mathcal{A}, \mathcal{B}, \Omega$, And preliminary Results}

We now make precise the assumptions on the nonlinearities $\mathcal{A}, \mathcal{B}$, and on the domain $\Omega$ that appear in equation (1.1). All of these assumptions will be needed in Theorem 3.1(ii) below.

Assumption 1. In (1.1), the nonlinearity $\mathcal{A}: \mathbb{R}^{n} \times \mathbb{R}^{n} \rightarrow \mathbb{R}^{n}$ is a Carathédory function, i.e., $\mathcal{A}(x, \xi)$ is measurable in $x$ for every $\xi$ and continuous in $\xi$ for a.e. $x \in \mathbb{R}^{n}$. Moreover, $\mathcal{A}(x, \xi)$ is continuously differentiable in $\xi$ away from the origin for a.e. $x \in \mathbb{R}^{n}$. We assume that for some $p>1$, it holds that

$$
\begin{gathered}
\langle\mathcal{A}(x, \xi)-\mathcal{A}(x, \eta), \xi-\eta\rangle \geq \Lambda_{0}\left(|\xi|^{2}+|\eta|^{2}\right)^{\frac{p-2}{2}}|\xi-\eta|^{2} \\
|\mathcal{A}(x, \xi)| \leq \Lambda_{1}|\xi|^{p-1}, \quad\left|\nabla_{\xi} \mathcal{A}(x, \zeta)\right| \leq \Lambda_{1}|\xi|^{p-2}
\end{gathered}
$$

for every $(\xi, \eta) \in \mathbb{R}^{n} \times \mathbb{R}^{n} \backslash(0,0)$ and a.e. $x \in \mathbb{R}^{n}$. Here $\Lambda_{0}$ and $\Lambda_{1}$ are positive constants.

Additionally, we suppose that $\mathcal{A}(x, \xi)$ satisfies the following $\left(\gamma, R_{0}\right)$-BMO condition in the $x$-variable, where $\gamma>0$ is sufficiently small.

Definition 2.1. Given two positive numbers $\gamma$ and $R_{0}$, we say that $\mathcal{A}(x, \xi)$ satisfies a $\left(\gamma, R_{0}\right)-B M O$ condition if

$$
\left.[\mathcal{A}]^{R_{0}}:=\sup _{y \in \mathbb{R}^{n}, 0<r \leq R_{0}} f_{B_{r}(y)} \Upsilon\left(\mathcal{A}, B_{r}(y)\right)\right)(x) d x \leq \gamma,
$$

where for a ball $B$ we set

$$
\Upsilon(\mathcal{A}, B)(x):=\sup _{\xi \in \mathbb{R}^{n} \backslash\{0\}} \frac{\left|\mathcal{A}(x, \xi)-\frac{1}{|B|} \int_{B} \mathcal{A}(y, \xi) d y\right|}{|\xi|^{p-1}} .
$$

Note that in the linear case, where $\mathcal{A}(x, \xi)=A(x) \xi$ for an elliptic matrix $A(x)$, we see that

$$
\Upsilon(\mathcal{A}, B)(x) \leq\left|A(x)-\frac{1}{|B|} \int_{B} A(y) d y\right|
$$


for a.e. $x \in \mathbb{R}^{n}$. Thus Definition 2.1 can be viewed as a natural extension of the standard small $\mathrm{BMO}$ condition to the nonlinear setting. We remark that the $\left(\gamma, R_{0}\right)$-BMO condition allows the nonlinearity $\mathcal{A}(x, \xi)$ to have certain discontinuity in $x$, and it can be used as an appropriate substitute for the Sarason VMO (vanishing mean oscillation) condition [32].

Assumption 2. In (1.1), the nonlinearity $\mathcal{B}: \Omega \times \mathbb{R} \times \mathbb{R}^{n} \rightarrow \mathbb{R}$ is a Carathédory function which satisfies, for a.e. $x \in \Omega$, every $s \in \mathbb{R}$, and every $\xi \in \mathbb{R}^{n}$,

$$
|\mathcal{B}(x, s, \xi)| \leq b_{0}|\xi|^{p}+b_{1}|s|^{m}, \quad \mathcal{B}(x, s, \xi) \operatorname{sign}(s) \leq b_{2}|\xi|^{p},
$$

where $m>p-1$, and $b_{0}, b_{1}, b_{2}$ are nonnegative constants.

Assumption 3. With regard to the underlying domain $\Omega$, we assume that its boundary is sufficiently flat in the sense of Reifenberg. This means essentially that at each boundary point and every scale, the boundary of $\Omega$ is trapped in between two hyperplanes separated by a distance proportional to the scale. Precisely, we assume that $\Omega$ is $\left(\gamma, R_{0}\right)$-Reifenberg flat for a sufficiently small $\gamma>0$. Below is the definition of a $\left(\gamma, R_{0}\right)$-Reifenberg flat domain.

Definition 2.2. Given $\gamma \in(0,1)$ and $R_{0}>0$, we say that $\Omega$ is $\left(\gamma, R_{0}\right)$-Reifenberg flat if for every $x_{0} \in \partial \Omega$ and every $r \in\left(0, R_{0}\right]$, there exists a system of coordinates $\left\{y_{1}, y_{2}, \ldots, y_{n}\right\}$, which may depend on $r$ and $x_{0}$, so that in this coordinate system $x_{0}=0$ and that

$$
B_{r}(0) \cap\left\{y_{n}>\gamma r\right\} \subset B_{r}(0) \cap \Omega \subset B_{r}(0) \cap\left\{y_{n}>-\gamma r\right\} .
$$

For more on Reifenberg flat domains and their many applications, we refer to the papers $[15,20,23,24,30,33]$. We mention here that Reifenberg flat domains can be very rough. They include Lipschitz domains with sufficiently small Lipschitz constants (see [33]) and even some domains with fractal boundaries. In particular, all bounded domains with $C^{1}$ boundaries are allowed in this paper.

Let $\mathbf{G}_{1} \mu$ be the first order Bessel's potential of a nonnegative locally finite measure $\mu$ defined by

$$
\mathbf{G}_{1} \mu(x)=\int_{\mathbb{R}^{n}} G_{1}(x-y) d \mu(y), \quad x \in \mathbb{R}^{n},
$$

where $G_{1}(x)$ is the Bessel kernel of order one defined via its Fourier transform by $\hat{G}_{1}(\xi)=$ $\left(1+|\xi|^{2}\right)^{-1 / 2}$.

Let $\operatorname{Cap}_{1, p}(\cdot)$ denote the capacity associated to the Sobolev space $W^{1, p}\left(\mathbb{R}^{n}\right)$, i.e.,

$$
\operatorname{Cap}_{1, p}(K):=\inf \left\{\int_{\mathbb{R}^{n}}\left(|\nabla \phi|^{p}+\varphi^{p}\right) d x: \phi \geq 1 \text { on } K, \quad 0 \leq \phi \leq 1, \quad \phi \in C_{c}^{\infty}\left(\mathbb{R}^{n}\right)\right\}
$$


for each compact set $K \subset \mathbb{R}^{n}$. It is well-known that $\operatorname{Cap}_{1, p}(\cdot)$ is equivalent to the Bessel capacity

$$
\operatorname{Cap}_{\mathbf{G}_{1}, p}(K):=\inf \left\{\int_{\mathbb{R}^{n}} f^{p} d x: f \in L^{p}\left(\mathbb{R}^{n}\right), f \geq 0, \text { and } \mathbf{G}_{1} f \geq 1 \text { on } K\right\} .
$$

We next recall a special case of Theorem 1.2 in [26]. This theorem enables us to reformulate the existence problem for (1.1) by means of the capacity $\operatorname{Cap}_{1, p}(\cdot)$.

Theorem 2.3. Let $\nu$ be a nonnegative locally finite measure in $\mathbb{R}^{n}$. Then the following properties of $\nu$ are equivalent.

(i) There is a constant $A_{1}>0$ such that

$$
\left.\int_{\mathbb{R}^{n}}|\varphi|^{p} d \nu \leq A_{1} \int_{\mathbb{R}^{n}}\left(|\nabla \varphi|^{p}\right)+|\varphi|^{p}\right) d x
$$

for all $\varphi \in C_{c}^{\infty}\left(\mathbb{R}^{n}\right)$.

(ii) There is a constant $A_{2}>0$ such that

$$
\int_{\mathbb{R}^{n}}\left(\mathbf{G}_{1} f\right)^{p} d \nu \leq A_{2} \int_{\mathbb{R}^{n}} f^{p} d x
$$

for all nonnegative $f \in L^{p}\left(\mathbb{R}^{n}\right)$.

(iii) There is a constant $A_{3}>0$ such that

$$
\nu(K) \leq A_{3} \operatorname{Cap}_{1, p}(K)
$$

for all compact sets $K \subset \mathbb{R}^{n}$.

(iv) There is a constant $A_{4}>0$ such that

$$
\int_{K}\left(\mathbf{G}_{1} \nu\right)^{\frac{p}{p-1}} d x \leq A_{4}^{\frac{p}{p-1}} \operatorname{Cap}_{1, p}(K)
$$

for all compact sets $K \subset \mathbb{R}^{n}$.

Moreover, the least possible values of the constants $A_{1}, A_{2}, A_{3}$, and $A_{4}$ are comparable to each other.

We now introduce a function space associated to the capacity $\operatorname{Cap}_{1, p}(\cdot)$, which plays a crucial role in our study of (1.1). This is the space $\mathrm{M}^{1, p}(\Omega)$ that was discussed in Section 1.

Definition 2.4. Let $\Omega \subset \mathbb{R}^{n}$ be an open set. We define $\mathrm{M}^{1, p}(\Omega)$ to be the set of all functions $f \in L^{p}(\Omega)$ such that there exists $C>0$ such that

$$
\int_{K}|f|^{p} d x \leq C \operatorname{Cap}_{1, p}(K)
$$


for all compact sets $K \subset \Omega$ The norm of $f \in \mathrm{M}^{1, p}(\Omega)$ is given by

$$
\|f\|_{\mathrm{M}^{1, p}(\Omega)}:=\sup _{K \subset \Omega}\left[\frac{\int_{K}|f|^{p} d x}{\operatorname{Cap}_{1, p}(K)}\right]^{\frac{1}{p}},
$$

where the sets $K$ vary over compact sets of $\Omega$ such that $\operatorname{Cap}_{1, p}(K)>0$.

Remark 2.5. For $f \in \mathrm{M}^{1, p}(\Omega)$, we will always implicitly extend $f$ by zero to $\mathbb{R}^{n} \backslash \Omega$, then inequality (2.4) actually holds for all compact sets $K \subset \mathbb{R}^{n}$. Thus by Theorem 2.3 we see that $f \in \mathrm{M}^{1, p}(\Omega)$ if and only if there exists $C>0$ such that

$$
\int_{\Omega}|\varphi|^{p}|f|^{p} d x \leq C \int_{\mathbb{R}^{n}}\left(|\nabla \varphi|^{p}+|\varphi|^{p}\right) d x
$$

for all $\varphi \in C_{c}^{\infty}\left(\mathbb{R}^{n}\right)$. That is, $f \in \mathrm{M}^{1, p}(\Omega)$ if and only if $f \chi_{\Omega} \in M\left(W^{1, p}\left(\mathbb{R}^{n}\right) \rightarrow L^{p}\left(\mathbb{R}^{n}\right)\right)$. Moreover, the best constants $C$ in (2.4) and (2.5) are equivalent, i.e., their ratio is bounded from above and below by positive constants independent of $f$. In particular, inequalities (1.6) and (1.7) simply mean that $|F|^{\frac{1}{p-1}}$ and $|\nabla u|$ belong to $\mathrm{M}^{1, p}(\Omega)$; and the best constants in (1.6) and (1.7) are equivalent to $\|\mid \nabla u\|_{\mathrm{M}^{1, p}(\Omega)}^{p}$ and $\left\||F|^{\frac{1}{p-1}}\right\|_{\mathrm{M}^{1, p}(\Omega)}^{p}$, respectively.

The following result will be useful to us.

Lemma 2.6. Suppose that $\mu$ is a finite sign measure in $\Omega$ such that

$$
|\mu|(K) \leq C_{1} \operatorname{Cap}_{1, p}(K)
$$

holds for all compact sets $K \subset \Omega$. Then we can write $\mu=\operatorname{div} F$ in $\mathcal{D}^{\prime}(\Omega)$ for a vector field $F$ such that $|F|^{\frac{1}{p-1}} \in \mathrm{M}^{1, p}(\Omega)$. Moreover,

$$
\left\||F|^{\frac{1}{p-1}}\right\|_{\mathrm{M}^{1, p}(\Omega)} \leq C(n, p, \operatorname{diam}(\Omega))\left(C_{1}\right)^{\frac{1}{p-1}} .
$$

Proof. After extending $\mu$ by zero outside $\Omega$, we may write $\mu=\operatorname{div} F$ in the sense of distributions in $\Omega$, where

$$
F(x)=-\int_{B} \nabla_{x} G(x, y) d \mu(y)
$$

Here $B$ is a ball of radius $\operatorname{diam}(\Omega)$ containing $\Omega$ and $G(x, y)$ is the Green function with zero boundary condition associated to $-\Delta$ on $B$. Note that we have

$$
\left|\nabla_{x} G(x, y)\right| \leq C|x-y|^{1-n} \leq C(n, \operatorname{diam}(\Omega)) G_{1}(x-y)
$$

for all $x, y \in B$ with $x \neq y$. Thus $|F(x)| \leq C \mathbf{G}_{1}(|\mu|)(x)$ which by Theorem (2.3) yields that $|F|^{\frac{1}{p-1}} \in \mathrm{M}^{1, p}(\Omega)$ along with estimate (2.7). Here note that as $|\mu|$ is zero outside $\Omega$, (2.6) actually holds for all compact sets $K \subset \mathbb{R}^{n}$. 
We now come to the key capacitary estimate that will make it possible to obtain a solution of (1.1) with strong regularity at the boundary of $\Omega$. This important estimate was the main motivation of our earlier work [2].

Lemma 2.7. Let $\mathcal{A}(x, \xi)$ and $\Omega$ satisfy Assumptions 1 and 3. That is, we assume $\mathcal{A}$ satisfies (2.1)-(2.2); $\mathcal{A}$ is $(\gamma, R)-B M O$; and $\Omega$ is $\left(\gamma, R_{0}\right)$-Reifenberg flat for a sufficiently small $\gamma=\gamma\left(n, p, \Lambda_{0}, \Lambda_{1}\right)>0$. Suppose that $F$ is a vector field such that $|F|^{\frac{1}{p-1}} \in \mathrm{M}^{1, p}(\Omega)$ and $u \in W_{0}^{1, p}(\Omega)$ is the unique solution of the equation

$$
\operatorname{div} \mathcal{A}(x, \nabla u)=\operatorname{div} F \text { in } \Omega, \quad u=0 \text { on } \partial \Omega .
$$

Then we have $|\nabla u| \in \mathrm{M}^{1, p}(\Omega)$ with

$$
\||\nabla u|\|_{\mathrm{M}^{1, p}(\Omega)} \leq C\left\||F|^{\frac{1}{p-1}}\right\|_{\mathrm{M}^{1, p}(\Omega)},
$$

where $C=C\left(n, p, \Lambda_{0}, \Lambda_{1}, \operatorname{diam}(\Omega) / R_{0}\right)$. Moreover, there exists a positive constant $C_{0}=$ $C_{0}(n, p, \operatorname{diam}(\Omega))$ such that for any $\mu \in\left(0, \mu_{0}\right]$ with

$$
\mu_{0}=\left(\Lambda_{0} / C_{0}\right)^{\frac{1}{p-1}}\left\||F|^{\frac{1}{p-1}}\right\|_{\mathrm{M}^{1, p}(\Omega)}^{-1},
$$

we have $e^{\mu|u|}-1 \in W_{0}^{1, p}(\Omega)$ with

$$
\left\|e^{\mu|u|}-1\right\|_{W_{0}^{1, p}(\Omega)} \leq C \mu\|F\|_{L^{\frac{p}{p-1}}(\Omega)}^{\frac{1}{p-1}},
$$

where $C=C\left(p, \Lambda_{0}\right)$.

Proof. Let $u \in W_{0}^{1, p}(\Omega)$ be the unique solution of (2.8). In [2], we showed that there exists $\gamma=\gamma\left(n, p, \Lambda_{0}, \Lambda_{1}\right)>0$ so that under Assumptions 1 and 3, the capacitary bound (2.9) holds with a constant $C=C\left(n, p, \Lambda_{0}, \Lambda_{1}\right.$, $\left.\operatorname{diam}(\Omega) / R_{0}\right)$; see [2, Corollary 1.8]. We mention that the proof of (2.9) is based on an end-point weighted estimate obtained in $[2$, Theorem 1.5] and a lemma of Verbitsky [26, Lemma 3.1]. In fact, the weighted estimate in $[2$, Theorem 1.5] was originally motivated from our study of (1.1). This is also where we need the $\left(\gamma, R_{0}\right)$-BMO condition on $\mathcal{A}$ and the $\left(\gamma, R_{0}\right)$-Reifenberg flatness condition on $\Omega$.

To verify (2.10), let $T_{s}, s>0$, denote the two-sided truncation operator at level $s$, i.e.,

$$
T_{s}(r)=r \text { if }|r| \leq s \text { and } T_{s}(r)=\operatorname{sign}(r) s \text { if }|r|>s .
$$

For $s, \mu>0$ we define

$$
u_{s}=T_{s}(u) \quad \text { and } \quad w_{s}=\operatorname{sign}(u)\left[e^{\mu\left|u_{s}\right|}-1\right] / \mu \text {, }
$$


where $\operatorname{sign}(u)=0$ if $u=0, \operatorname{sign}(u)=1$ if $u>0$, and $\operatorname{sign}(u)=-1$ if $u<0$.

Note that we have $\nabla w_{s}=e^{\mu\left|u_{s}\right|} \nabla u_{s}=\left(e^{\mu\left|u_{s}\right|} \nabla u\right) \chi_{\{|u| \leq s\}}$ and thus if we let

$$
v_{s}=e^{\delta\left|u_{s}\right|} w_{s}, \quad \delta=(p-1) \mu,
$$

then it holds that

$$
\begin{aligned}
\nabla v_{s} & =\left[e^{\delta\left|u_{s}\right|} \nabla w_{s}+\delta\left|w_{s}\right| e^{\delta\left|u_{s}\right|} \nabla u\right] \chi_{\{|u| \leq s\}} \\
& =\left[e^{p \mu\left|u_{s}\right|} \nabla u+\delta\left|w_{s}\right| e^{\delta\left|u_{s}\right|} \nabla u\right] \chi_{\{|u| \leq s\}},
\end{aligned}
$$

since $\mu+\delta=p \mu$.

Using $v_{s}$ as a test function in (2.8) and employing (2.12), we get

$$
\begin{aligned}
& \int_{\Omega} \mathcal{A}(x, \nabla u) \cdot \nabla u e^{p \mu\left|u_{s}\right|} \chi_{\{|u| \leq s\}} d x \\
& \quad=-\int_{\Omega} \delta\left|w_{s}\right| e^{\delta\left|u_{s}\right|} \mathcal{A}(x, \nabla u) \cdot \nabla u \chi_{\{|u| \leq s\}} d x+\int_{\Omega} F \cdot \nabla v_{s} d x .
\end{aligned}
$$

By (2.1)-(2.2) the first term on the right-hand side is nonpositive, and thus we get

$$
\int_{\Omega} \mathcal{A}(x, \nabla u) \cdot \nabla u e^{p \mu\left|u_{s}\right|} \chi_{\{|u| \leq s\}} d x \leq \int_{\Omega} F \cdot \nabla v_{s} d x .
$$

Since $\nabla w_{s}=e^{\mu\left|u_{s}\right|} \nabla u_{s}$, using conditions (2.1)-(2.2), we see that

$$
\begin{aligned}
\int_{\Omega} \mathcal{A}(x, \nabla u) \cdot \nabla u e^{p \mu\left|u_{s}\right|} \chi_{\{|u| \leq s\}} d x & \geq \Lambda_{0} \int_{\Omega}|\nabla u|^{p} e^{p \mu\left|u_{s}\right|} \chi_{\{|u| \leq s\}} d x \\
& =\Lambda_{0} \int_{\Omega}\left|\nabla w_{s}\right|^{p} d x .
\end{aligned}
$$

On the other hand, as $v_{s}=e^{\delta\left|u_{s}\right|} w_{s}=\left(1+\mu\left|w_{s}\right|\right)^{p-1} w_{s}$ we have

$$
\begin{aligned}
\int_{\Omega} F \cdot \nabla v_{s} d x= & \int_{\Omega} F \cdot \nabla\left[\left(1+\mu\left|w_{s}\right|\right)^{p-1} w_{s}\right] d x \\
= & \int_{\Omega} F \cdot\left[(p-1)\left(1+\mu\left|w_{s}\right|\right)^{p-2} \nabla w_{s} \operatorname{sign}\left(w_{s}\right) \mu w_{s}\right] d x+ \\
& +\int_{\Omega} F \cdot\left[\left(1+\mu\left|w_{s}\right|\right)^{p-1} \nabla w_{s}\right] d x \\
\leq & p \int_{\Omega}|F|\left(1+\mu\left|w_{s}\right|\right)^{p-1}\left|\nabla w_{s}\right| d x
\end{aligned}
$$

Using the inequality

$$
\left(1+\mu\left|w_{s}\right|\right)^{p-1} \leq 2 \mu^{p-1}\left|w_{s}\right|^{p-1}+C(p),
$$

and Hölder's inequality in the above bound we then have 


$$
\begin{aligned}
\int_{\Omega} F \cdot \nabla v_{s} d x \leq & 2 \mu^{p-1} p \int_{\Omega}|F|\left|w_{s}\right|^{p-1}\left|\nabla w_{s}\right| d x+C(p)\|F\|_{L^{\frac{p}{p-1}}(\Omega)}\left\|\nabla w_{s}\right\|_{L^{p}(\Omega)} \\
\leq & 2 \mu^{p-1} p\left(\int_{\Omega}|F|^{\frac{p}{p-1}}\left|w_{s}\right|^{p} d x\right)^{\frac{p-1}{p}}\left\|\nabla w_{s}\right\|_{L^{p}(\Omega)} \\
& +C(p)\|F\|_{L^{\frac{p}{p-1}(\Omega)}}\left\|\nabla w_{s}\right\|_{L^{p}(\Omega)} .
\end{aligned}
$$

Note that by assumption $|F|^{\frac{1}{p-1}} \in \mathrm{M}^{1, p}(\Omega)$, Theorem 2.3, and Poincaré's inequality we have

$$
\begin{aligned}
\left(\int_{\Omega}|F|^{\frac{p}{p-1}}\left|w_{s}\right|^{p} d x\right)^{\frac{p-1}{p}} & \leq C\left\||F|^{\frac{1}{p-1}}\right\|_{\mathrm{M}^{1, p}(\Omega)}^{p-1}\left[\int_{\Omega}\left(\left|\nabla w_{s}\right|^{p}+\left|w_{s}\right|^{p}\right) d x\right]^{\frac{p-1}{p}} \\
& \leq C(n, p, \operatorname{diam}(\Omega))\left\||F|^{\frac{1}{p-1}}\right\|_{\mathrm{M}^{1, p}(\Omega)}^{p-1}\left(\int_{\Omega}\left|\nabla w_{s}\right|^{p} d x\right)^{\frac{p-1}{p}} .
\end{aligned}
$$

This gives

$$
\begin{aligned}
\int_{\Omega} F \cdot \nabla v_{s} d x \leq & C_{1} \mu^{p-1}\left\||F|^{\frac{1}{p-1}}\right\|_{\mathrm{M}^{1, p}(\Omega)}^{p-1}\left\|\nabla w_{s}\right\|_{L^{p}(\Omega)}^{p} \\
& +C(p)\|F\|_{L^{\frac{p}{p-1}}(\Omega)}\left\|\nabla w_{s}\right\|_{L^{p}(\Omega)},
\end{aligned}
$$

where $C_{1}=C_{1}(n, p, \operatorname{diam}(\Omega))$. At this point we combine estimates (2.14) and (2.15) in equality (2.13) to obtain the following bound

$$
\left(\Lambda_{0}-C_{1} \mu^{p-1}\left\||F|^{\frac{1}{p-1}}\right\|_{M^{1, p}(\Omega)}^{p-1}\right)\left\|\nabla w_{s}\right\|_{L^{p}(\Omega)}^{p-1} \leq C(p)\|F\|_{L^{\frac{p}{p-1}}(\Omega)} .
$$

This gives

$$
\left\|\nabla w_{s}\right\|_{L^{p}(\Omega)} \leq C(p)\left(\Lambda_{0} / 2\right)^{\frac{-1}{p-1}}\|F\|_{L^{\frac{p}{p-1}}(\Omega)}^{\frac{1}{p-1}}
$$

provided

$$
\mu \leq\left(\frac{\Lambda_{0}}{2 C_{1}}\right)^{\frac{1}{p-1}}\left\||F|^{\frac{1}{p-1}}\right\|_{\mathrm{M}^{1, p}(\Omega)}^{-1} .
$$

Finally, letting $s \nearrow+\infty$ we obtain the desiblack estimate in $W_{0}^{1, p}(\Omega)$ for $e^{\mu|u|}-1$.

The following convergence result, shown in [5], will be important for us in the proof of Theorem 3.1(ii).

Theorem 2.8 ([5]). Suppose that $\mathcal{A}$ satisfies (2.1)-(2.2). For each $k>0$, let $w_{k} \in W^{1, p}(\Omega)$ be a solution to the equation

$$
-\operatorname{div} \mathcal{A}\left(x, \nabla w_{k}\right)=m_{k}+h_{k} \quad \text { in } \mathcal{D}^{\prime}(\Omega)
$$

and assume that 
- $w_{k} \rightarrow w$ weakly in $W^{1, p}(\Omega)$, strongly in $L_{\mathrm{loc}}^{p}(\Omega)$, and a.e. in $\Omega$;

- $h_{k} \rightarrow h$ in $\left(W_{0}^{1, p}(\Omega)\right)^{*}$;

- $m_{k}$ is bounded in the space of finite Radon measures in $\Omega$. That is, $\left|\left\langle m_{k}, \phi\right\rangle\right| \leq$ $C_{K}\|\phi\|_{L^{\infty}(\Omega)}$ for all $\phi \in C_{c}^{\infty}(\Omega)$ with $\operatorname{spt}(\phi) \subset K$, where $C_{K}$ depends on $K$ but not on $k$.

Then it holds that $\nabla w_{k} \rightarrow \nabla w$ in $L^{q}(\Omega)$ for all $q<p$, and thus up to a subsequence $\nabla w_{k} \rightarrow \nabla w$ a.e. in $\Omega$.

We will also need the following strong convergence result first proved in F. E. Browder [7] (see also [6, Lemma 5]).

Lemma 2.9. Under (2.1)-(2.2), assume that the following two hypotheses are satisfied:

$$
\begin{gathered}
u_{\varepsilon} \rightarrow u \quad \text { in } W_{0}^{1, p}(\Omega) \text { weakly and a.e. in } \Omega, \\
\int_{\Omega}\left[\mathcal{A}\left(x, \nabla u_{\varepsilon}\right)-\mathcal{A}(x, \nabla u)\right] \cdot \nabla\left(u_{\varepsilon}-u\right) d x \rightarrow 0 .
\end{gathered}
$$

Then it holds that

$$
u_{\varepsilon} \rightarrow u \quad \text { in } W_{0}^{1, p}(\Omega) \text { strongly. }
$$

\section{Equations With General Structures and main RESUlts}

We are now ready to state the main result of the paper regarding the existence theory for equation (1.1) in the space $\mathrm{M}^{1, p}(\Omega)$. From our discussion on $\mathrm{M}^{1, p}(\Omega)$, we see that Theorem 1.1 is just a special case of the following more general result.

Theorem 3.1. (i) Suppose that $\mathcal{A}(x, \xi)$ satisfies the first inequality in $(2.2)$, and $\mathcal{B}(x, s, \xi)$ satisfies the first inequality (2.3). If equation (1.1) has a solution $u \in W_{0}^{1, p}(\Omega)$ with $|\nabla u| \in$ $\mathrm{M}^{1, p}(\Omega)$, then there exists a vector field $F$ such that $\sigma=\operatorname{div} F$ and $|F|^{\frac{1}{p-1}} \in \mathrm{M}^{1, p}(\Omega)$ satisfying the estimate

$$
\left\||F|^{\frac{1}{p-1}}\right\|_{\mathrm{M}^{1, p}(\Omega)} \leq C\left\{\|\nabla u\|_{\mathrm{M}^{1, p}(\Omega)}+\|\nabla u\|_{\mathrm{M}^{1, p}(\Omega)}^{\frac{p}{p-1}}+\|\nabla u\|_{\mathrm{M}^{1, p}(\Omega)}^{\frac{m}{p-1}}\right\} .
$$

(ii) Let $\mathcal{A}, \mathcal{B}$, and $\Omega$ satisfy Assumptions 1, 2, and 3. That is, we assume $\mathcal{A}, \mathcal{B}$ satisfy (2.1)-(2.3); $\mathcal{A}$ is $(\gamma, R)$-BMO; and $\Omega$ is $\left(\gamma, R_{0}\right)$-Reifenberg flat for a sufficiently small $\gamma=$ $\gamma\left(n, p, \Lambda_{0}, \Lambda_{1}\right)>0$. Suppose that $\sigma=\operatorname{div} F$ for a vector field $F$ such that $|F|^{\frac{1}{p-1}} \in \mathrm{M}^{1, p}(\Omega)$. There is a positive number $\lambda_{0}=\lambda_{0}\left(n, p, m, \Lambda_{0}, \Lambda_{1}, b_{0}, b_{1}, b_{2}\right.$, $\left.\operatorname{diam}(\Omega), R_{0}\right)>0$ such that if

$$
\left\||F|^{\frac{1}{p-1}}\right\|_{\mathrm{M}^{1, p}(\Omega)} \leq \lambda_{0}^{1 / p}
$$


then equation (1.1) has a solution $u \in W_{0}^{1, p}(\Omega)$ with $|\nabla u| \in \mathrm{M}^{1, p}(\Omega)$ and

$$
\||\nabla u|\|_{\mathrm{M}^{1, p}(\Omega)} \leq T_{0}\left\||F|^{\frac{1}{p-1}}\right\|_{\mathrm{M}^{1, p}(\Omega)} .
$$

Moreover, $u$ satisfies $e^{\mu|u|}-1 \in W_{0}^{1, p}(\Omega)$ for all $\mu \in\left[0, \mu_{0}\right]$ where $\mu_{0}=C\left\||F|^{\frac{1}{p-1}}\right\|_{M^{1, p}(\Omega)}^{-1}$, and

$$
\left\|e^{\mu|u|}-1\right\|_{W_{0}^{1, p}(\Omega)} \leq C\left(p, \Lambda_{0}\right) \mu\|F\|_{L^{\frac{p}{p-1}}(\Omega)}^{\frac{1}{p-1}} .
$$

We now devote to the proof of Theorem 3.1. We first start with part (i):

Proof of Theorem 3.1(i). Let $u \in W_{0}^{1, p}(\Omega)$ be such that $|\nabla u| \in \mathrm{M}^{1, p}(\Omega)$. Then for $F=|\nabla u|^{p-2} \nabla u$, we have that $u$ solves

$$
\Delta_{p} u=\operatorname{div} F \text { in } \Omega, \quad u=0 \text { on } \partial \Omega .
$$

Thus by Lemma 2.7 we have $e^{\mu_{0}|u|}-1 \in W_{0}^{1, p}(\Omega)$ with

$$
\left\|e^{\mu_{0}|u|}-1\right\|_{W_{0}^{1, p}(\Omega)} \leq C(p) \mu_{0}\|F\|_{L^{\frac{p}{p-1}}(\Omega)}^{\frac{1}{p-1}},
$$

where

$$
\mu_{0}=c(n, p, \operatorname{diam}(\Omega))\left\||F|^{\frac{1}{p-1}}\right\|_{\mathrm{M}^{1, p}(\Omega)}^{-1}=c(n, p, \operatorname{diam}(\Omega))\||\nabla u|\|_{\mathrm{M}^{1, p}(\Omega)}^{-1} .
$$

Note that

$$
\|F\|_{L^{\frac{p}{p-1}}(\Omega)}^{\frac{1}{p-1}}=\|\nabla u\|_{L^{p}(\Omega)} \leq C(n, p, \operatorname{diam}(\Omega))\|\mid \nabla u\|_{\mathrm{M}^{1, p}(\Omega)},
$$

and thus we get

$$
\left\|e^{\mu_{0}|u|}-1\right\|_{W_{0}^{1, p}(\Omega)} \leq C(n, p, \operatorname{diam}(\Omega)) .
$$

On the other hand, for any $m_{0}$ such that $m m_{0} \geq 1$, we have

$$
\left|\mu_{0} u\right|^{m m_{0}} \leq\left\lceil m m_{0}\right\rceil !\left(e^{\mu_{0}|u|}-1\right)
$$

where $\lceil x\rceil$ denotes the smallest integer larger than or equal to $x$. Hence, by Poincaré's inequality and (3.3) we find

$$
\int_{\Omega}\left|\mu_{0} u\right|^{m m_{0}} d x \leq C\left\|e^{\mu_{0}|u|}-1\right\|_{W_{0}^{1, p}(\Omega)} \leq C .
$$

That is, we have

$$
\left(\int_{\Omega}|u|^{m m_{0}} d x\right)^{\frac{1}{m m_{0}}} \leq C \mu_{0}^{-1} \leq C\||\nabla u|\|_{\mathrm{M}^{1, p}(\Omega)}
$$


by (3.2). Moreover, by Hölder's inequality we see that (3.4) in fact holds for all $m_{0}>0$ with a constant $C=C\left(n, p, m, m_{0}\right.$, $\left.\operatorname{diam}(\Omega)\right)$. Thus for $m_{0}>1$, using Hölder's inequality we get

$$
\int_{K}|u|^{m} d x \leq\left\||u|^{m}\right\|_{L^{m_{0}(\Omega)}}|K|^{1-\frac{1}{m_{0}}} \leq C\left\|\left.\left|\nabla u \|_{\mathrm{M}^{1, p}(\Omega)}^{m}\right| K\right|^{1-\frac{1}{m_{0}}}\right.
$$

for any compact set $K \subset \Omega$.

We next define $\kappa=n /(n-p)$ if $1<p<n$ and $\kappa=2$ if $p \geq n$. Then by Sobolev's inequality, for any compact set $K$ we have

$$
|K|^{\frac{1}{\kappa}} \leq\left(\int_{\mathbb{R}^{n}} \varphi^{\kappa p} d x\right)^{\frac{1}{\kappa}} \leq C \int_{\mathbb{R}^{n}}\left(|\nabla \varphi|^{p}+\varphi^{p}\right) d x
$$

for all $\varphi \in C_{c}^{\infty}\left(\mathbb{R}^{n}\right), 0 \leq \varphi \leq 1$ and $\varphi=1$ on $K$. This gives $|K|^{\frac{1}{\kappa}} \leq C \operatorname{Cap}_{1, p}(K)$ and thus choosing $m_{0}$ in (3.5) so that $1-1 / m_{0}=1 / \kappa$ we get

$$
\int_{K}|u|^{m} d x \leq C\||\nabla u|\|_{\mathrm{M}^{1, p}(\Omega)}^{m} \operatorname{Cap}_{1, p}(K) .
$$

Now using (3.6), the first bound in (2.3), and the fact that $|\nabla u| \in \mathrm{M}^{1, p}(\Omega)$ we have

$$
\int_{K}|\mathcal{B}(x, u, \nabla u)| d x \leq\left\{b_{0}\||\nabla u|\|_{\mathrm{M}^{1, p}(\Omega)}^{p}+C b_{1}\||\nabla u|\|_{\mathrm{M}^{1, p}(\Omega)}^{m}\right\} \operatorname{Cap}_{1, p}(K)
$$

for all compact sets $K \subset \Omega$. Thus by Lemma 2.6 we can write $\mathcal{B}(x, u, \nabla u)=\operatorname{div} F_{1}$ for a vector field $F_{1}$ such that

$$
\left\|\left|F_{1}\right|^{\frac{1}{p-1}}\right\|_{\mathrm{M}^{1, p}(\Omega)} \leq C\||\nabla u|\|_{\mathrm{M}^{1, p}(\Omega)}^{\frac{p}{p-1}}+C\||\nabla u|\|_{\mathrm{M}^{1, p}(\Omega)}^{\frac{m}{p-1}} .
$$

Now assume in addition that $u$ is a solution of (1.1), then we have

$$
\sigma=-\operatorname{div} \mathcal{A}(x, \nabla u)-\mathcal{B}(x, u, \nabla u)=\operatorname{div}\left[-\mathcal{A}(x, \nabla u)-F_{1}\right] .
$$

Thus letting $F=-\mathcal{A}(x, \nabla u)-F_{1}$ and using the first bound in (2.2), we get the desiblack result.

We next prove part (ii) of Theorem 3.1.

Proof of Theorem 3.1(ii). The proof of this part will be carried out in several steps. First, we approximate (1.1) and then obtain existence and regularity for the approximate equation. Eventually, we will use the regularity and appropriate test functions to pass to the limit.

We begin by setting, for each $T>0$,

$$
\mathbb{E}_{T}:=\left\{\phi \in W_{0}^{1,1}(\Omega): \phi \in W_{0}^{1, p}(\Omega) \text { and }\||\nabla \phi|\|_{\mathrm{M}^{1, p}(\Omega)} \leq T\left\||F|^{\frac{1}{p-1}}\right\|_{\mathrm{M}^{1, p}(\Omega)}\right\} .
$$


We shall impose the subset topology from $W_{0}^{1,1}(\Omega)$ on the set $\mathbb{E}_{T}$. In fact, we could also use in $E_{T}$ the strong topology of $W_{0}^{1, q}(\Omega)$ for any $1<q<p$. However, there is a problem with compactness that prevents us from using the natural topology of $W_{0}^{1, p}(\Omega)$ for $E_{T}$.

It is easy to see from the definition of $\mathbb{E}_{T}$ and Fatou's lemma that $E_{T}$ is convex and closed under the strong topology of $W_{0}^{1,1}(\Omega)$.

For $k>0$, we now define a function $\mathcal{H}_{k}(x, s, \xi)$ by letting

$$
\mathcal{H}_{k}(x, s, \xi):=\frac{\mathcal{B}(x, s, \xi)}{1+|\mathcal{B}(x, s, \xi)| k^{-1}} .
$$

Then $\mathcal{H}_{k}(x, s, \xi)$ also satisfies $(2.3)$ and $\left|\mathcal{H}_{k}(x, s, \xi)\right| \leq \min \{k,|\mathcal{B}(x, s, \xi)|\}$. For each $v \in \mathbb{E}_{T}$ and each vector field $F$ such that $|F|^{\frac{1}{p-1}} \in \mathrm{M}^{1, p}(\Omega)$ we let $S(v)$ denote the unique solution $u \in W_{0}^{1, p}(\Omega)$ to the equation

$$
-\operatorname{div} \mathcal{A}(x, \nabla u)=\mathcal{H}_{k}(x, v, \nabla v)+\operatorname{div} F \text { in } \Omega, \quad u=0 \text { on } \partial \Omega .
$$

The map $S: \mathbb{E}_{T} \rightarrow W_{0}^{1, p}(\Omega)$ is well defined as the right-hand side of the first equation in (3.8) belongs to $\left(W_{0}^{1, p}(\Omega)\right)^{*}$. Note that we have not yet made any choice on $T$. We shall break the proof into three steps.

Step 1. In this step we show that there exists $T_{0}>0$ independent of $k$ such that whenever

$$
\left\||F|^{\frac{1}{p-1}}\right\|_{\mathrm{M}^{1, p}(\Omega)} \leq 2 \min \left\{\left(2 T_{0}\right)^{-p},\left(2 T_{0}\right)^{\frac{-m}{m-p+1}}\right\}
$$

we have

$$
S: \mathbb{E}_{T_{0}} \rightarrow \mathbb{E}_{T_{0}}
$$

Indeed, since $\left|\mathcal{H}_{k}(x, v, \nabla v)\right| \leq|\mathcal{B}(x, v, \nabla v)|$, by calculations as in the proof of part (i) (see $(3.7))$ we have

$$
\int_{K}\left|\mathcal{H}_{k}(x, v, \nabla v)\right| d x \leq\left\{b_{0}\||\nabla v|\|_{\mathrm{M}^{1, p}(\Omega)}^{p}+C b_{1}\||\nabla v|\|_{\mathrm{M}^{1, p}(\Omega)}^{m}\right\} \operatorname{Cap}_{1, p}(K)
$$

for any compact set $K$. Thus by Lemma 2.6 we can write $\mathcal{H}_{k}(x, v, \nabla v)=\operatorname{div} F_{k}$ for a vector field $F_{k}$ such that

$$
\left\|\left|F_{k}\right|^{\frac{1}{p-1}}\right\|_{\mathrm{M}^{1, p}(\Omega)} \leq C\left\{\||\nabla v|\|_{\mathrm{M}^{1, p}(\Omega)}^{\frac{p}{p-1}}+\||\nabla v|\|_{\mathrm{M}^{1, p}(\Omega)}^{\frac{m}{p-1}}\right\} .
$$

Then from Lemma 2.7 we find that $|\nabla S(v)| \in \mathrm{M}^{1, p}(\Omega)$ with

$$
\begin{aligned}
\||\nabla S(v)|\|_{\mathrm{M}^{1, p}(\Omega)} & \leq C\left\|\left|F_{k}+F\right|^{\frac{1}{p-1}}\right\|_{\mathrm{M}^{1, p}(\Omega)} \\
& \leq \bar{C}\left\{\||\nabla v|\|_{\mathrm{M}^{1, p}(\Omega)}^{\frac{p}{p-1}}+\||\nabla v|\|_{\mathrm{M}^{1, p}(\Omega)}^{\frac{m}{p-1}}+\left\||F|^{\frac{1}{p-1}}\right\|_{\mathrm{M}^{1, p}(\Omega)}\right\}
\end{aligned}
$$


for a constant $\bar{C}$ independent of $k$.

We now choose $T_{0}=2 \bar{C}$. Then for $v \in \mathbb{E}_{T_{0}}$ and $F$ satisfying (3.9) we have

$$
\begin{aligned}
\||\nabla S(v)|\|_{\mathrm{M}^{1, p}(\Omega)} \leq & \bar{C}\left(T_{0}^{\frac{p}{p-1}}\left\||F|^{\frac{1}{p-1}}\right\|_{\mathrm{M}^{1, p}(\Omega)}^{\frac{1}{p-1}}+T_{0}^{\frac{m}{p-1}}\left\||F|^{\frac{1}{p-1}}\right\|_{\mathrm{M}^{1, p}(\Omega)}^{\frac{m-p+1}{p-1}}\right)\left\||F|^{\frac{1}{p-1}}\right\|_{\mathrm{M}^{1, p}(\Omega)} \\
& +\bar{C}\left\||F|^{\frac{1}{p-1}}\right\|_{\mathrm{M}^{1, p}(\Omega)} \\
\leq & 2 \bar{C}\left\||F|^{\frac{1}{p-1}}\right\|_{\mathrm{M}^{1, p}(\Omega)}=T_{0}\left\||F|^{\frac{1}{p-1}}\right\|_{\mathrm{M}^{1, p}(\Omega)} .
\end{aligned}
$$

This gives $S(v) \in \mathbb{E}_{T_{0}}$ and thus (3.10) follows.

Step 2. We now prove that for each $k>0$ there exists a solution $u_{k} \in \mathbb{E}_{T_{0}}$ to the approximate equation

$$
-\operatorname{div} \mathcal{A}\left(x, \nabla u_{k}\right)=\mathcal{H}_{k}\left(x, u_{k}, \nabla u_{k}\right)+\operatorname{div} F \text { in } \Omega, \quad u_{k}=0 \text { on } \partial \Omega .
$$

To that end, we shall use Schauder's Fixed Point Theorem to obtain a fixed point for the map $S: \mathbb{E}_{T_{0}} \rightarrow \mathbb{E}_{T_{0}}$. Since we already know that $\mathbb{E}_{T_{0}}$ is closed and convex, it remains to show that $S: \mathbb{E}_{T_{0}} \rightarrow \mathbb{E}_{T_{0}}$ is continuous and $S\left(\mathbb{E}_{T_{0}}\right)$ is pre-compact (under the strong topology of $\left.W_{0}^{1,1}(\Omega)\right)$.

To prove continuity, let $\left\{v_{l}\right\} \subset \mathbb{E}_{T_{0}}$ be a sequence such that $v_{l} \rightarrow v$ strongly in $W_{0}^{1,1}(\Omega)$. This combined with the fact that $\left\{S\left(v_{l}\right)\right\}$ is bounded in $W_{0}^{1, p}(\Omega)$, there is a subsequence, also denoted by $\left\{v_{l}\right\}$ for simplicity, and a function $u \in W_{0}^{1, p}(\Omega)$ such that:

(a) $S\left(v_{l}\right) \rightarrow u$ weakly in $W_{0}^{1, p}(\Omega)$, strongly in $L^{p}(\Omega)$, and a.e. in $\Omega$,

(b) $\left\{\mathcal{H}_{k}\left(x, v_{l}, \nabla v_{l}\right)\right\}_{l}$ is uniformly bounded in the space of finite signed measures in $\Omega$ for each fixed $k>0$.

Recall that we have

$$
-\operatorname{div} \mathcal{A}\left(x, \nabla\left[S\left(v_{l}\right)\right]\right)=\mathcal{H}_{k}\left(x, v_{l}, \nabla v_{l}\right)+\operatorname{div} F \quad \text { in } \mathcal{D}^{\prime}(\Omega) .
$$

Thus by Theorem 2.8 we have $S\left(v_{l}\right) \rightarrow u$ in $W_{0}^{1, q}(\Omega)$ for any $1 \leq q<p$, and up to another subsequence we have $\nabla\left[S\left(v_{l}\right)\right] \rightarrow \nabla u$ a.e. in $\Omega$.

By (2.2) and Vitali's Convergence Theorem we have

$$
\mathcal{A}\left(x, \nabla\left[S\left(v_{l}\right)\right]\right) \rightarrow \mathcal{A}(x, \nabla u) \quad \text { strongly in } L^{1}\left(\Omega, \mathbb{R}^{n}\right) \text { and weakly in } L^{p /(p-1)}\left(\Omega, \mathbb{R}^{n}\right) .
$$

Up to another subsequence, it holds that $v_{l} \rightarrow v$ and $\nabla v_{l} \rightarrow \nabla v$ a.e. in $\Omega$. Thus by Dominated Convergence Theorem we have

$$
\mathcal{H}_{k}\left(x, v_{l}, \nabla v_{l}\right) \rightarrow \mathcal{H}_{k}(x, v, \nabla v) \quad \text { in } L^{1}(\Omega)
$$


This is where we use the property that $\left|\mathcal{H}_{k}\left(x, v_{l}, \nabla v_{l}\right)\right| \leq k$ for all $l$.

Thus we can pass to the limit in equation (3.12) to obtain that $u=S(v)$. So far we have found a subsequence of $\left\{v_{l_{j}}\right\}$ of $\left\{v_{l}\right\}$ such that $S\left(v_{l_{j}}\right) \rightarrow S(v)$ in $W_{0}^{1,1}(\Omega)$. As the limit is independent of the subsequence it actually holds that the whole sequence $S\left(v_{l}\right) \rightarrow S(v)$ in $W_{0}^{1,1}(\Omega)$. This shows that the map $S: \mathbb{E}_{T_{0}} \rightarrow \mathbb{E}_{T_{0}}$ is continuous.

To prove pre-compactness, let $\left\{u_{l}\right\}=\left\{S\left(v_{l}\right)\right\}$ be a sequence in $S\left(\mathbb{E}_{T_{0}}\right)$, where $v_{l} \in \mathbb{E}_{T_{0}}$. Then as above there is a subsequence of $\left\{v_{l}\right\}$, also denoted by $\left\{v_{l}\right\}$, and a function $u \in$ $W_{0}^{1, p}(\Omega)$ such that properties (a) and (b) above hold. Thus by Theorem 2.8 again we have $S\left(v_{l}\right) \rightarrow u$ in $W_{0}^{1,1}(\Omega)$. This shows that the set $S\left(\mathbb{E}_{T_{0}}\right)$ is pre-compact.

Step 3. In this step we further restrict that

$$
\left\||F|^{\frac{1}{p-1}}\right\|_{\mathrm{M}^{1, p}(\Omega)} \leq \lambda_{0}^{\frac{1}{p}}
$$

where

$$
\lambda_{0}^{\frac{1}{p}}:=\min \left\{2 \min \left\{\left(2 T_{0}\right)^{-p},\left(2 T_{0}\right)^{\frac{-m}{m-p+1}}\right\},\left(b_{2} / \Lambda_{0}\right)^{-1}\left(\Lambda_{0} / C_{0}\right)^{\frac{1}{p-1}}\right\},
$$

and $C_{0}=C_{0}(n, p, \operatorname{diam}(\Omega))$ is as in Lemma 2.7. Then we have

$$
\mu_{0}=\left(\Lambda_{0} / C_{0}\right)^{\frac{1}{p-1}}\left\|\mid F^{\frac{1}{p-1}}\right\|_{\mathrm{M}^{1, p}(\Omega)}^{-1} \geq b_{2} / \Lambda_{0}
$$

Let $u_{k}$ be as in Step 2. By Lemma 2.7, we have $e^{\mu\left|u_{k}\right|}-1 \in W_{0}^{1, p}(\Omega)$ for $\mu \in\left[0, \mu_{0}\right]$, with

$$
\left\|e^{\mu\left|u_{k}\right|}-1\right\|_{W_{0}^{1, p}(\Omega)} \leq C \mu\|F\|_{L^{\frac{p}{p-1}}(\Omega)}^{\frac{1}{p-1}}
$$

where $C=C\left(p, \Lambda_{0}\right)$. Then by Rellich's compactness theorem, there is a subsequence, still denoted by $\left\{u_{k}\right\}$, such that

$$
u_{k} \stackrel{k}{\rightarrow} u \text { weakly in } W_{0}^{1, p}(\Omega), \text { strongly in } L^{p}(\Omega), \text { and a.e. in } \Omega,
$$

for a function $u \in W_{0}^{1, p}(\Omega)$ such that (3.1) holds for all $\mu \in\left[0, \mu_{0}\right]$.

We now claim that

$$
u_{k} \rightarrow u \text { strongly in } W_{0}^{1, p}(\Omega) \text { as } k \nearrow \infty
$$

and thus we can pass to the limit in (3.11) to verify that $u$ is a solution to (1.1).

To prove (3.15), we write

$$
\nabla u_{k}-\nabla u=\nabla T_{s}\left(u_{k}\right)-\nabla T_{s}(u)+\nabla G_{s}\left(u_{k}\right)-\nabla G_{s}(u)
$$


where $s>0$ and $G_{s}(r):=r-T_{s}(r), r \in \mathbb{R}$, and $T_{s}$ is as defined in (2.11). Thus for every $s>0$ we have

$$
\begin{aligned}
\left\|\nabla u_{k}-\nabla u\right\|_{L^{p}(\Omega)} \leq & \sup _{k>0}\left\|\nabla G_{s}\left(u_{k}\right)-\nabla G_{s}(u)\right\|_{L^{p}(\Omega)} \\
& +\left\|\nabla T_{s}\left(u_{k}\right)-\nabla T_{s}(u)\right\|_{L^{p}(\Omega)} .
\end{aligned}
$$

Note that for $\mu \in\left(0, \mu_{0}\right]$ we find

$$
\begin{aligned}
\int_{\Omega}\left|\nabla G_{s}\left(u_{k}\right)\right|^{p} d x & =\int_{\left\{\left|u_{k}\right|>s\right\}}\left|\nabla u_{k}\right|^{p} d x \\
& =\frac{1}{\mu} \int_{\left\{\left|u_{k}\right|>s\right\}} e^{-p \mu\left|u_{k}\right|}\left|\nabla\left(e^{\mu\left|u_{k}\right|}-1\right)\right|^{p} d x \\
& \leq \frac{1}{\mu} e^{-p \mu s}\left\|e^{\mu\left|u_{k}\right|}-1\right\|_{W_{0}^{1, p}(\Omega)}^{p} .
\end{aligned}
$$

By (3.14), this yields that

$$
\lim _{s \rightarrow \infty} \sup _{k>0}\left\|\nabla G_{s}\left(u_{k}\right)-\nabla G_{s}(u)\right\|_{L^{p}(\Omega)}=0 .
$$

On the other hand, by (3.13) and Lemma 3.2 below it holds that

$$
\lim _{k \rightarrow \infty}\left\|\nabla T_{s}\left(u_{k}\right)-\nabla T_{s}(u)\right\|_{L^{p}(\Omega)}=0 \quad \text { for each } s>0 .
$$

Thus combining (3.16), (3.17), and (3.18) we obtain convergence (3.15) as desiblack. This completes the proof of the theorem.

We are now left with the proof of the following lemma.

Lemma 3.2. Let $\mathcal{A}, \mathcal{B}$ satisfy $(2.1)-(2.3)$ and let $F$ be a vector field in $L^{\frac{p}{p-1}}\left(\Omega, \mathbb{R}^{n}\right)$. For each $k>0$, let $u_{k} \in W_{0}^{1, p}(\Omega)$ be a solution of (3.11). Suppose that there exists $\mu_{0} \geq b_{2} / \Lambda_{0}$ such that $\left\{e^{\mu_{0}\left|u_{k}\right|}-1\right\}$ is uniformly bounded in $W_{0}^{1, p}(\Omega)$. Suppose also that

$$
u_{k} \stackrel{k}{\rightarrow} u \text { weakly in } W_{0}^{1, p}(\Omega), \text { strongly in } L^{p}(\Omega) \text {, and a.e. in } \Omega \text {, }
$$

for a function $u \in W_{0}^{1, p}(\Omega)$. Then we have the convergence (3.18) for any $s>0$.

Proof. Let $s>0$ be fixed. For any $j \geq s$ we define

$$
v_{k}=e^{\mu_{0}\left|T_{j}\left(u_{k}\right)\right|} \psi\left(z_{k}\right),
$$

where $z_{k}=T_{s}\left(u_{k}\right)-T_{s}(u)$ and $\psi$ is a $C^{1}$ and increasing function from $\mathbb{R}$ to $\mathbb{R}$ satisfying

$$
\psi(0)=0 \quad \text { and } \quad \psi^{\prime}-\frac{b_{0}+\Lambda_{1} \mu_{0}}{\Lambda_{0}}|\psi| \geq 1 .
$$


As in [12] (see also $[4,13])$, using $v_{k}$ test function in (3.11), we have

$$
\begin{aligned}
\int_{\Omega} & \mathcal{A}\left(x, \nabla u_{k}\right) \cdot e^{\mu_{0}\left|T_{j}\left(u_{k}\right)\right|} \psi^{\prime}\left(z_{k}\right) \nabla z_{k} d x \\
= & \int_{\Omega}\left[\mathcal{H}_{k}\left(x, u_{k}, \nabla u_{k}\right)-\mu_{0} \mathcal{A}\left(x, \nabla u_{k}\right) \cdot \nabla T_{j}\left(u_{k}\right) \operatorname{sign}\left(u_{k}\right)\right] e^{\mu_{0}\left|T_{j}\left(u_{k}\right)\right|} \psi\left(z_{k}\right) d x \\
& \quad+\int_{\Omega} F \cdot \nabla\left[e^{\mu_{0}\left|T_{j}\left(u_{k}\right)\right|} \psi\left(z_{k}\right)\right] d x .
\end{aligned}
$$

Note that the term on the left-hand side in the above equality can be written as

$$
\begin{aligned}
& \int_{\Omega} \mathcal{A}\left(x, \nabla u_{k}\right) \cdot\left(\nabla T_{s}\left(u_{k}\right)-\nabla T_{s}(u)\right) e^{\mu_{0}\left|T_{j}\left(u_{k}\right)\right|} \psi^{\prime}\left(z_{k}\right) d x \\
& =\int_{\left\{\left|u_{k}\right| \leq s\right\}}\left\{\mathcal{A}\left(x, \nabla T_{s}\left(u_{k}\right)\right)-\mathcal{A}\left(x, \nabla T_{s}(u)\right)\right\} \cdot \\
& \quad \cdot\left(\nabla T_{s}\left(u_{k}\right)-\nabla T_{s}(u)\right) e^{\mu_{0}\left|T_{j}\left(u_{k}\right)\right|} \psi^{\prime}\left(z_{k}\right) d x \\
& \quad+\int_{\left\{\left|u_{k}\right| \leq s\right\}} \mathcal{A}\left(x, \nabla T_{s}(u)\right) \cdot\left(\nabla T_{s}\left(u_{k}\right)-\nabla T_{s}(u)\right) e^{\mu_{0}\left|T_{j}\left(u_{k}\right)\right|} \psi^{\prime}\left(z_{k}\right) d x \\
& \quad+\int_{\left\{\left|u_{k}\right|>s\right\}} \mathcal{A}\left(x, \nabla u_{k}\right) \cdot\left(-\nabla T_{s}(u)\right) e^{\mu_{0}\left|T_{j}\left(u_{k}\right)\right|} \psi^{\prime}\left(z_{k}\right) d x
\end{aligned}
$$

Thus combining the last two equalities we obtain

$$
I_{1}-I_{4}=-I_{2}-I_{3}+I_{5}
$$

where we define that

$$
\begin{gathered}
I_{1}=\int_{\left\{\left|u_{k}\right| \leq s\right\}}\left\{\mathcal{A}\left(x, \nabla T_{s}\left(u_{k}\right)\right)-\mathcal{A}\left(x, \nabla T_{s}(u)\right)\right\} \cdot \\
\cdot\left(\nabla T_{s}\left(u_{k}\right)-\nabla T_{s}(u)\right) e^{\mu_{0}\left|T_{j}\left(u_{k}\right)\right|} \psi^{\prime}\left(z_{k}\right) d x \\
I_{2}=\int_{\left\{\left|u_{k}\right| \leq s\right\}} \mathcal{A}\left(x, \nabla T_{s}(u)\right) \cdot\left(\nabla T_{s}\left(u_{k}\right)-\nabla T_{s}(u)\right) e^{\mu_{0}\left|T_{j}\left(u_{k}\right)\right|} \psi^{\prime}\left(z_{k}\right) d x \\
I_{3}=\int_{\left\{\left|u_{k}\right|>s\right\}} \mathcal{A}\left(x, \nabla u_{k}\right) \cdot\left(-\nabla T_{s}(u)\right) e^{\mu_{0}\left|T_{j}\left(u_{k}\right)\right|} \psi^{\prime}\left(z_{k}\right) d x \\
I_{4}=\int_{\Omega}\left[\mathcal{H}_{k}\left(x, u_{k}, \nabla u_{k}\right)-\mu_{0} \mathcal{A}\left(x, \nabla u_{k}\right) \cdot \nabla T_{j}\left(u_{k}\right) \operatorname{sign}\left(u_{k}\right)\right] e^{\mu_{0}\left|T_{j}\left(u_{k}\right)\right|} \psi\left(z_{k}\right) d x,
\end{gathered}
$$

and

We further have

$$
I_{5}=\int_{\Omega} F \cdot \nabla\left[e^{\mu_{0}\left|T_{j}\left(u_{k}\right)\right|} \psi\left(z_{k}\right)\right] d x
$$

where

$$
I_{4}^{1}:=\int_{\left\{\left|u_{k}\right|>s\right\}}\{\ldots\} d x, \quad I_{4}^{2}:=\int_{\left\{\left|u_{k}\right| \leq s\right\}}\{\ldots\} d x
$$


with $\{\ldots\}$ being the integrand in $I_{4}$.

Since $\left|\nabla T_{j}\left(u_{k}\right)\right| \leq\left|\nabla u_{k}\right|$, using (2.1)-(2.3) we get

$$
\begin{aligned}
\left|I_{4}^{2}\right| \leq & \int_{\left\{\left|u_{k}\right| \leq s\right\}}\left(b_{0}\left|\nabla u_{k}\right|^{p}+b_{1}\left|u_{k}\right|^{m}+\mu_{0} \Lambda_{1}\left|\nabla u_{k}\right|^{p}\right) e^{\mu_{0}\left|T_{j}\left(u_{k}\right)\right|}\left|\psi\left(z_{k}\right)\right| d x \\
\leq & \frac{b_{0}+\mu_{0} \Lambda_{1}}{\Lambda_{0}} \int_{\left\{\left|u_{k}\right| \leq s\right\}} \mathcal{A}\left(x, \nabla T_{s}\left(u_{k}\right)\right) \cdot \nabla T_{s}\left(u_{k}\right) e^{\mu_{0}\left|T_{j}\left(u_{k}\right)\right|}\left|\psi\left(z_{k}\right)\right| d x \\
& +\int_{\left\{\left|u_{k}\right| \leq s\right\}} b_{1} s^{m} e^{\mu_{0} s}\left|\psi\left(z_{k}\right)\right| d x,
\end{aligned}
$$

Thus, with $M=\left(b_{0}+\mu_{0} \Lambda_{1}\right) / \Lambda_{0}$, we find that

$$
\begin{aligned}
\left|I_{4}^{2}\right| \leq & M \int_{\left\{\left|u_{k}\right| \leq s\right\}}\left[\mathcal{A}\left(x, \nabla T_{s}\left(u_{k}\right)\right)-\mathcal{A}\left(x, \nabla T_{s}(u)\right)\right] \cdot \\
& \cdot\left[\nabla T_{s}\left(u_{k}\right)-\nabla T_{s}(u)\right] e^{\mu_{0}\left|T_{j}\left(u_{k}\right)\right|}\left|\psi\left(z_{k}\right)\right| d x \\
& +M \int_{\left\{\left|u_{k}\right| \leq s\right\}} \mathcal{A}\left(x, \nabla T_{s}(u)\right) \cdot\left[\nabla T_{s}\left(u_{k}\right)-\nabla T_{s}(u)\right] e^{\mu_{0}\left|T_{j}\left(u_{k}\right)\right|}\left|\psi\left(z_{k}\right)\right| d x \\
& +M \int_{\left\{\left|u_{k}\right| \leq s\right\}} \mathcal{A}\left(x, \nabla T_{s}\left(u_{k}\right)\right) \cdot \nabla T_{s}(u) e^{\mu_{0}\left|T_{j}\left(u_{k}\right)\right|}\left|\psi\left(z_{k}\right)\right| d x \\
& +\int_{\left\{\left|u_{k}\right| \leq s\right\}} b_{1} s^{m} e^{\mu_{0} s}\left|\psi\left(z_{k}\right)\right| d x .
\end{aligned}
$$

On the other hand, using the inequalities

$$
\mathcal{A}\left(x, \nabla u_{k}\right) \cdot \nabla T_{j}\left(u_{k}\right) \geq \Lambda_{0}\left|\nabla u_{k}\right|^{p} \chi_{\left\{\left|u_{k}\right| \leq j\right\}}, \quad \chi_{\left\{\left|u_{k}\right|>s\right\}} \operatorname{sign}\left(u_{k}\right) \psi\left(z_{k}\right) \geq 0,
$$

and (2.3), we have

$$
\begin{aligned}
I_{4}^{1}= & \int_{\left\{\left|u_{k}\right|>s\right\}}\left[\operatorname{sign}\left(u_{k}\right) \mathcal{H}_{k}\left(x, u_{k}, \nabla u_{k}\right)-\mu_{0} \mathcal{A}\left(x, \nabla u_{k}\right) \cdot \nabla T_{j}\left(u_{k}\right)\right] \times \\
& \times \operatorname{sign}\left(u_{k}\right) e^{\mu_{0}\left|T_{j}\left(u_{k}\right)\right|} \psi\left(z_{k}\right) d x \\
\leq & \int_{\left\{\left|u_{k}\right|>s\right\}}\left[b_{2}\left|\nabla u_{k}\right|^{p}-\mu_{0} \Lambda_{0}\left|\nabla u_{k}\right|^{p} \chi_{\left\{\left|u_{k}\right| \leq j\right\}}\right] \operatorname{sign}\left(u_{k}\right) e^{\mu_{0}\left|T_{j}\left(u_{k}\right)\right|} \psi\left(z_{k}\right) d x .
\end{aligned}
$$

Thus since $\mu_{0} \Lambda_{0} \geq b_{2}$ and $j \geq s$, we get

$$
\begin{aligned}
I_{4}^{1} & \leq \int_{\left\{\left|u_{k}\right|>j\right\}} b_{2}\left|\nabla u_{k}\right|^{p} \operatorname{sign}\left(u_{k}\right) e^{\mu_{0}\left|T_{j}\left(u_{k}\right)\right|} \psi\left(z_{k}\right) d x \\
& \leq b_{2} \max _{r \in[-2 s, 2 s]}|\psi(r)| e^{\mu_{0} j} \int_{\left\{\left|u_{k}\right|>j\right\}}\left|\nabla u_{k}\right|^{p} d x \\
& \leq C e^{\mu_{0} j} \frac{1}{\mu_{0}} \int_{\left\{\left|u_{k}\right|>j\right\}} e^{-p \mu_{0}\left|u_{k}\right|}\left|\nabla\left(e^{\mu_{0}\left|u_{k}\right|}-1\right)\right|^{p} d x \\
& \leq C \frac{1}{\mu_{0}} e^{\mu_{0} j} e^{-p \mu_{0} j}\left\|e^{\mu_{0}\left|u_{k}\right|}-1\right\|_{W_{0}^{1, p}(\Omega)}^{p} .
\end{aligned}
$$


As the sequence $\left\{e^{\mu_{0}\left|u_{k}\right|}-1\right\}$ is uniformly bounded in $W_{0}^{1, p}(\Omega)$, this yields that

$$
\limsup _{j \rightarrow \infty} \sup _{k>0} I_{4}^{1} \leq 0 .
$$

Let $D_{k}$ be the nonnegative function

$$
D_{k}:=\left(\mathcal{A}\left(x, \nabla T_{s}\left(u_{k}\right)\right)-\mathcal{A}\left(x, \nabla T_{s}(u)\right)\right) \cdot\left(\nabla T_{s}\left(u_{k}\right)-\nabla T_{s}(u)\right) .
$$

Then by (3.19),

$$
\int_{\left\{\left|u_{k}\right| \leq s\right\}} D_{k} d x \leq \int_{\left\{\left|u_{k}\right| \leq s\right\}} D_{k} e^{\mu_{0}\left|T_{j}\left(u_{k}\right)\right|}\left(\psi^{\prime}-M|\psi|\right) d x .
$$

Thus combining this with (3.21)-(3.22), we get

$$
\begin{aligned}
& \int_{\left\{\left|u_{k}\right| \leq s\right\}} D_{k} d x \leq I_{1}-I_{4}+ \\
& \quad+M \int_{\left\{\left|u_{k}\right| \leq s\right\}} \mathcal{A}\left(x, \nabla T_{s}(u)\right) \cdot\left[\nabla T_{s}\left(u_{k}\right)-\nabla T_{s}(u)\right] e^{\mu_{0}\left|T_{j}\left(u_{k}\right)\right|}\left|\psi\left(z_{k}\right)\right| d x \\
& \quad+M \int_{\left\{\left|u_{k}\right| \leq s\right\}} \mathcal{A}\left(x, \nabla T_{s}\left(u_{k}\right)\right) \cdot \nabla T_{s}(u) e^{\mu_{0}\left|T_{j}\left(u_{k}\right)\right|}\left|\psi\left(z_{k}\right)\right| d x \\
& \quad+\int_{\left\{\left|u_{k}\right| \leq s\right\}} b_{1} s^{m} e^{\mu_{0} s}\left|\psi\left(z_{k}\right)\right| d x+\varepsilon
\end{aligned}
$$

for any $\varepsilon>0$ provided $j=j(\varepsilon)$ is sufficiently large.

Our next goal is to apply $\lim \sup _{k \rightarrow \infty}$ to both sides of the above inequality. To that end, note that $\psi(0)=0, z_{k} \stackrel{k}{\rightarrow} 0$ a.e., $\left\{\nabla T_{s}\left(u_{k}\right)-\nabla T_{s}(u)\right\}$ and $\left\{\mathcal{A}\left(x, \nabla T_{s}\left(u_{k}\right)\right)\right\}$ are uniformly bounded in $L^{p}\left(\Omega, \mathbb{R}^{n}\right)$ and in $L^{p /(p-1)}\left(\Omega, \mathbb{R}^{n}\right)$, respectively. Thus by Hölder's inequality and Dominated Convergence Theorem we find

$$
\begin{gathered}
\lim _{k \rightarrow \infty} \int_{\left\{\left|u_{k}\right| \leq s\right\}} \mathcal{A}\left(x, \nabla T_{s}(u)\right) \cdot\left[\nabla T_{s}\left(u_{k}\right)-\nabla T_{s}(u)\right] e^{\mu_{0}\left|T_{j}\left(u_{k}\right)\right|}\left|\psi\left(z_{k}\right)\right| d x, \\
\lim _{k \rightarrow \infty} \int_{\left\{\left|u_{k}\right| \leq s\right\}} \mathcal{A}\left(x, \nabla T_{s}\left(u_{k}\right)\right) \cdot \nabla T_{s}(u) e^{\mu_{0}\left|T_{j}\left(u_{k}\right)\right|}\left|\psi\left(z_{k}\right)\right| d x=0,
\end{gathered}
$$

and

$$
\lim _{k \rightarrow \infty} \int_{\left\{\left|u_{k}\right| \leq s\right\}} b_{1}|s|^{m} e^{\mu_{0} s}\left|\psi\left(z_{k}\right)\right| d x=0 .
$$

Thus we get

$$
\begin{aligned}
\limsup _{k \rightarrow \infty} \int_{\left\{\left|u_{k}\right| \leq s\right\}} D_{k} d x & \leq \limsup _{k \rightarrow \infty}\left(I_{1}-I_{4}\right)+\varepsilon \\
& =\limsup _{k \rightarrow \infty}\left(-I_{2}-I_{3}+I_{5}\right)+\varepsilon
\end{aligned}
$$

for any $\varepsilon>0$ provided $j=j(\varepsilon)$ is sufficiently large. Here we use (3.20) in the last equality. 
We next claim that for any $j \geq s$ we have

$$
\lim _{k \rightarrow \infty}\left(-I_{2}-I_{3}+I_{5}\right)=0 .
$$

To prove this claim, we treat each term on the right-hand side separately as follows.

The term $I_{2}$ : Since $u_{k} \stackrel{k}{\rightarrow} u$ a.e. and $z_{k} \stackrel{k}{\rightarrow} 0$ a.e., it holds that

$$
\mathcal{A}\left(x, \nabla T_{s}(u)\right) e^{\mu_{0}\left|T_{j}\left(u_{k}\right)\right|} \psi^{\prime}\left(z_{k}\right) \stackrel{k}{\rightarrow} \mathcal{A}\left(x, \nabla T_{s}(u)\right) e^{\mu_{0}\left|T_{j}(u)\right|} \psi^{\prime}(0) \quad \text { a.e. }
$$

Thus using the pointwise estimate,

$$
\left|\mathcal{A}\left(x, \nabla T_{s}(u)\right) e^{\mu_{0}\left|T_{j}\left(u_{k}\right)\right|} \psi^{\prime}\left(z_{k}\right)\right| \leq e^{\mu_{0} j} \max _{r \in[-2 s, 2 s]}\left|\psi^{\prime}(r)\right| \Lambda_{1}\left|\nabla T_{s}(u)\right|^{p-1}
$$

and Dominated Convergence Theorem, we have

$$
\mathcal{A}\left(x, \nabla T_{s}(u)\right) e^{\mu_{0}\left|T_{j}\left(u_{k}\right)\right|} \psi^{\prime}\left(z_{k}\right) \stackrel{k}{\rightarrow} \mathcal{A}\left(x, \nabla T_{s}(u)\right) e^{\mu_{0}\left|T_{j}(u)\right|} \psi^{\prime}(0)
$$

strongly in $L^{p /(p-1)}\left(\Omega, \mathbb{R}^{n}\right)$.

Next, since $u_{k}$ is uniformly bounded in $W_{0}^{1, p}(\Omega)$ and $T_{s}\left(u_{k}\right) \stackrel{k}{\rightarrow} T_{s}(u)$ a.e., we have $\nabla T_{s}\left(u_{k}\right) \stackrel{k}{\longrightarrow} \nabla T_{s}(u)$ weakly in $L^{p}\left(\Omega, \mathbb{R}^{n}\right)$. On the other hand, since

$$
\chi_{\left\{\left|u_{k}\right| \leq s\right\}} \stackrel{k}{\rightarrow} \chi_{\{|u| \leq s\}} \text { a.e. in } \Omega \backslash\{|u|=s\} \text { and }\left|\nabla T_{s}(u)\right|=0 \text { a.e. on }\{|u|=s\},
$$

we have from Dominated Convergence Theorem that

$$
\nabla T_{s}(u) \chi_{\left\{\left|u_{k}\right| \leq s\right\}} \stackrel{k}{\rightarrow} \nabla T_{s}(u) \chi_{\{|u| \leq s\}}=\nabla T_{s}(u) \quad \text { strongly in } \quad L^{p}\left(\Omega, \mathbb{R}^{n}\right) .
$$

Thus,

$$
\begin{aligned}
\chi_{\left\{\left|u_{k}\right| \leq s\right\}}\left(\nabla T_{s}\left(u_{k}\right)-\nabla T_{s}(u)\right) & =\nabla T_{s}\left(u_{k}\right)-\nabla T_{s}(u) \chi_{\left\{\left|u_{k}\right| \leq s\right\}} \\
& \stackrel{k}{\longrightarrow} 0 \text { weakly in } L^{p}\left(\Omega, \mathbb{R}^{n}\right) .
\end{aligned}
$$

These convergences imply that

$$
\lim _{k \rightarrow \infty} I_{2}=0
$$

The term $I_{3}$ : By $(2.2),\left|\mathcal{A}\left(x, \nabla u_{k}\right)\right|$ is uniformly bounded in $L^{p /(p-1)}(\Omega)$. On the other hand, again by (3.25) and Dominated Convergence Theorem we have

$$
\left|\chi_{\left\{\left|u_{k}\right|>s\right\}}\left(-\nabla T_{s}(u)\right) e^{\mu_{0}\left|T_{j}\left(u_{k}\right)\right|} \psi^{\prime}\left(z_{k}\right)\right| \stackrel{k}{\rightarrow} 0 \quad \text { strongly in } \quad L^{p}(\Omega) .
$$

Thus using Hölder's inequality we see that

$$
\lim _{k \rightarrow \infty} I_{3}=0
$$


The term $I_{5}$ : We have

$$
\begin{aligned}
I_{5}= & \mu_{0} \int_{\Omega} F \cdot e^{\mu_{0}\left|T_{j}\left(u_{k}\right)\right|} \psi\left(z_{k}\right) \nabla T_{j}\left(u_{k}\right) \operatorname{sign}\left(u_{k}\right) d x \\
& +\int_{\Omega} F \cdot e^{\mu_{0}\left|T_{j}\left(u_{k}\right)\right|} \psi^{\prime}\left(z_{k}\right) \nabla z_{k} d x .
\end{aligned}
$$

As $F e^{\mu_{0}\left|T_{j}\left(u_{k}\right)\right|} \psi\left(z_{k}\right) \stackrel{k}{\rightarrow}(0, \ldots, 0)$ a.e. in $\Omega$, by Dominated Convergence Theorem we find

$$
F e^{\mu_{0}\left|T_{j}\left(u_{k}\right)\right|} \psi\left(z_{k}\right) \stackrel{k}{\rightarrow}(0, \ldots, 0) \quad \text { strongly in } L^{p /(p-1)}\left(\Omega, \mathbb{R}^{n}\right) .
$$

Since $\nabla T_{j}\left(u_{k}\right) \operatorname{sign}\left(u_{k}\right)$ is uniformly bounded in $L^{p}\left(\Omega, \mathbb{R}^{n}\right)$, we then conclude that

$$
\mu_{0} \int_{\Omega} F \cdot e^{\mu_{0}\left|T_{j}\left(u_{k}\right)\right|} \psi\left(z_{k}\right) \nabla T_{j}\left(u_{k}\right) \operatorname{sign}\left(u_{k}\right) d x \stackrel{k}{\rightarrow} 0 .
$$

Again, by Dominated Convergence Theorem we have

$$
F e^{\mu_{0}\left|T_{j}\left(u_{k}\right)\right|} \psi^{\prime}\left(z_{k}\right) \stackrel{k}{\rightarrow} F e^{\delta\left|T_{j}(u)\right|} \psi^{\prime}(0) \quad \text { strongly in } \quad L^{p /(p-1)}\left(\Omega, \mathbb{R}^{n}\right) .
$$

Thus using (3.26) and $\nabla z_{k}=\nabla T_{s}\left(u_{k}\right)-\nabla T_{s}(u)$, we obtain that

$$
\int_{\left\{\left|u_{k}\right| \leq s\right\}} F \cdot e^{\mu_{0}\left|T_{j}\left(u_{k}\right)\right|} \psi^{\prime}\left(z_{k}\right) \nabla z_{k} d x \stackrel{k}{\rightarrow} 0 .
$$

On the other hand,

$$
\begin{aligned}
& \int_{\left\{\left|u_{k}\right|>s\right\}} F \cdot e^{\mu_{0}\left|T_{j}\left(u_{k}\right)\right|} \psi^{\prime}\left(z_{k}\right) \nabla z_{k} d x \\
& \quad=\int_{\Omega} F \cdot e^{\mu_{0}\left|T_{j}\left(u_{k}\right)\right|} \psi^{\prime}\left(z_{k}\right)\left(-\nabla T_{s}(u)\right) \chi_{\left\{\left|u_{k}\right|>s\right\}} d x \\
& \quad \stackrel{k}{\rightarrow} 0
\end{aligned}
$$

by (3.25), Hölder's inequality, and Dominated Convergence Theorem.

Combining the last two limits, we obtain

$$
\int_{\Omega} F \cdot e^{\mu_{0}\left|T_{j}\left(u_{k}\right)\right|} \psi^{\prime}\left(z_{k}\right) \nabla z_{k} d x \stackrel{k}{\rightarrow} 0 .
$$

Hence combining (3.27), (3.28), and (3.29), we conclude that

$$
\lim _{k \rightarrow \infty} I_{5}=0
$$

Thus we have shown that the limit (3.24) holds. Then in view of (3.23) and the fact that $D_{k} \geq 0$ we find that

$$
\int_{\left\{\left|u_{k}\right| \leq s\right\}} D_{k} d x \stackrel{k}{\rightarrow} 0
$$


On the other hand, by (3.25),

$$
\begin{aligned}
\chi_{\left\{\left|u_{k}\right|>s\right\}} D_{k} & =\chi_{\left\{\left|u_{k}\right|>s\right\}}\left[\mathcal{A}(x, 0)-\mathcal{A}\left(x, \nabla T_{s}(u)\right)\right] \cdot\left(-\nabla T_{s}(u)\right) \\
& \stackrel{k}{\rightarrow} 0 \quad \text { a.e. }
\end{aligned}
$$

which implies that $\int_{\left\{\left|u_{k}\right|>s\right\}} D_{k} d x \stackrel{k}{\rightarrow} 0$. Thus we obtain

$$
\int_{\Omega} D_{k} d x \stackrel{k}{\rightarrow} 0
$$

Finally, with (3.30) we can apply Lemma 2.9 to conclude the proof of (3.18) as desiblack.

\section{REFERENCES}

[1] B. Abdellaoui, A. Dall'Aglio, and I. Peral, Some remarks on elliptic problems with critical growth in the gradient, J. Differential Equations 222 (2006) 21-62.

[2] K. Adimurthi and N. C. Phuc, An end-point global gradient weighted estimate for quasilinear equations in non-smooth domains, Manuscripta Math. 150 (2016), 111-135.

[3] K. Adimurthi and N. C. Phuc, Nonlinear equations with gradient natural growth and distributional data, with applications to a Schrödinger type equation, preprint 2017.

[4] A. Bensoussan, L. Boccardo, and F. Murat, On a nonlinear partial differential equation having natural growth terms and unbounded solution, Ann. Inst. H. Poincaré Anal. Non Linéaire, 5 (1988), 347-364.

[5] L. Boccardo and F. Murat, Almost everywhere convergence of the gradients of solutions to elliptic and parabolic equations, Nonlinear Anal. 19 (1992), 581-597.

[6] L. Boccardo, F. Murat and J.-P. Puel, Existence of bounded solutions for nonlinear elliptic unilateral problems, Ann. Mat. Pura Appl. (4) 152 (1988), 183-196.

[7] F. E. Browder, Existence theorems for nonlinear partial differential equations, in S.-S. Chern, S. Smale (Eds), Proc. Sympos. Pure Math., Vol. XVI, pp. 1-60, Amer. Math. Soc., Providence, R.I. 1970.

[8] S.-Y. A. Chang, J. M. Wilson, and T. H. Wolff, Some weighted norm inequalities concerning the Schrödinger operators, Comment. Math. Helv. 60 (1985), 217-246.

[9] D. M. Duc, N. C. Phuc, and T. V. Nguyen, Weighted Sobolev's inequalities for bounded domains and singular elliptic equations, Indiana Univ. Math. J. 56 (2007), 615-642.

[10] C. Fefferman, The uncertainty principle, Bull. Amer. Math. Soc. 9 (1983), 129-206.

[11] V. Ferone and F. Murat, Quasilinear problems having natural growth in the gradient: an existence result when the source term is small, in: Équations aux dérivées partielles et applications, Articles dédiés à Jacques-Louis Lions, Gauthier-Villars, Paris, 1998, pp. 497-515.

[12] V. Ferone and F. Murat, Nonlinear problems having natural growth in the gradient: an existence result when the source terms are small, Nonlinear Anal., 42 (2000), 1309-1326.

[13] V. Ferone and F. Murat, Nonlinear elliptic equations with natural growth in the gradient and source terms in Lorentz Spaces, J. Differential Equations 256 (2014), 577-608. 
[14] M. Frazier and I. E. Verbitsky, Positive solutions to Schrdinger's equation and the exponential integrability of the balayage, Preprint 2015, arXiv:1509.09005.

[15] P. Hajlasz and O. Martio, Traces of Sobolev functions on fractal type sets and characterization of extension domains, J. Funct. Anal. 143 (1997) 221-246.

[16] H. A. Hamid and M. F. Bidaut-Veron, On the connection between two quasilinear elliptic problems with source terms of order 0 or 1 . Commun. Contemp. Math. 12 (2010), 727-788.

[17] K. Hansson, V. G. Maz'ya, and I. E. Verbitsky, Criteria of solvability for multidimensional Riccati equations, Ark. Mat. 37 (1999), 87-120.

[18] B. Jaye, V. G. Maz'ya, and I. E. Verbitsky, Existence and regularity of positive solutions of elliptic equations of Schrdinger type, J. Anal. Math. 118 (2012), 577-621.

[19] B. Jaye, V. G. Maz'ya, and I. E. Verbitsky, Quasilinear elliptic equations and weighted Sobolev-Poincaré inequalities with distributional weights, Adv. Math. 232 (2013), 513-542.

[20] P. W. Jones, Quasiconformal mappings and extendability of functions in Sobolev spaces, Acta Math. 147 (1981) 71-88.

[21] M. Kardar, G. Parisi, and Y.-C. Zhang, Dynamic scaling of growing interfaces, Phys. Rev. Lett. 56 (1986) 889-892.

[22] J. Krug and H. Spohn, Universality classes for deterministic surface growth, Phys. Rev. A (3) 38 (1988) 4271-4283.

[23] C. Kenig and T. Toro, Free boundary regularity for harmonic measures and the Poisson kernel, Ann. Math. 150 (1999) 367-454.

[24] C. Kenig and T. Toro, Poisson kernel characterization of Reifenberg flat chord arc domains, Ann. Sci. École Norm. Sup. (4) 36 (2003) 323-401.

[25] J.-L. Lions, Quelques méthodes de résolution des problèmes aux limites non linéaires, Dunod; GauthierVillars, Paris 1969. xx+554 pp.

[26] V. G. Maz'ya and I. E. Verbitsky, Capacitary inequalities for fractional integrals, with applications to partial differential equations and Sobolev multipliers, Ark. Math. 33 (1995), 81-115.

[27] V. G. Maz'ya and T. O. Shaposhnikova, The theory of Sobolev multipliers with applications to differential and integral operators, Grundlehren der math. Wissenschaften, vol. 337, Springer, 2009.

[28] T. Mengesha and N. C. Phuc, Quasilinear Ricatti type equations with distributional data in Morrey space framework, J. Differential Equations 260 (2016), 5421-5449.

[29] C. Pérez, Two weighted inequalities for potential and fractional type maximal operators, Indiana Univ. Math. J. 43 (1994), 663-683.

[30] E. Reifenberg, Solutions of the Plateau Problem for m-dimensional surfaces of varying topological type, Acta Math. 104 (1960) 1-92.

[31] E. T. Sawyer and R. L. Wheeden, Weighted inequalities for fractional integrals on Euclidean and homogeneous spaces, Amer. J. Math. 114 (1992), 813-874.

[32] D. Sarason, Functions of vanishing mean oscillation, Trans. Amer. Math. Soc. 207 (1975), 391-405.

[33] T. Toro, Doubling and flatness: geometry of measures, Notices Amer. Math. Soc. 44 (1997) $1087-1094$. 
${ }^{1}$ Department of Mathematical Sciences, Seoul National University, GwanAkRo 1, GwanakGu, Seoul 08826, South Korea.

E-mail address: kadimurthi@snu.ac.kr and karthikaditi@gmail.com

2 Department of Mathematics, Louisiana State University, 303 Lockett Hall, Baton Rouge, LA 70803, USA.

E-mail address: pcnguyen@math.1su.edu 